\title{
Le deuil dans Muettes de Yasmine Ghata ${ }^{1}$
}

\section{Aliaa Ahmed Abdel Wahed*}

aliawahed@yahoo.com

\section{Résumé}

Le deuil est une expérience douloureuse consécutive à la perte d'un être cher. Cette perte provoque un chagrin qui s'en nourrit. Dès lors, la personne endeuillée a besoin de la consolation de son entourage, ainsi que d'un grand soutien collectif pour surmonter sa souffrance et alléger son traumatisme.

Dans le roman de Yasmine Ghata, la narratrice, une fillette de 6 ans, et sa mère perdent l'une son père, l'autre son conjoint. Une période de deuil est inéluctable pour toutes les deux. Malgré le choc du décès, elles refusent les condoléances et la consolation d'autrui, préférant souffrir dans le silence et le mutisme.

Cependant, comment surmonter la souffrance liée à un deuil, comment se réjouir à nouveau sans appui ni consolation d'autrui ?

L'étude est scindée en trois parties: le deuil inconsolé, le deuil scriptural et le deuil mémoriel.

La première partie analyse la façon dont la mère et sa fille se comportent sans consolation, en affrontant le choc du décès. La conclusion en est que la vie devient maussade et n'a plus de sens. Mère et fille sont menacées de traumatismes si elles ne parviennent pas à vivre cette période de façon apaisée.

La seconde partie étudie la stratégie adoptée par la mère afin de s'approprier le deuil: le recours à l'écriture. Cependant, l'univers scriptural demeure un monde lugubre où morts et cimetières s'emparent du récit. Les mots reflètent le vide et l'absence. En outre, l'écriture est un remède éphémère: lorsque le récit s'achève, il faut affronter la réalité et l'absence. L'écriture devient donc endeuillée, ou un prolongement du deuil.

* Professeur adjoint en littérature francaise et francophone - faculté des lettres et sciences humaines- Université Canal de Suez.

(Le deuil dans Muettes de Yasmine Ghata ...) Dr. Aliaa Abdel Wahed 
La troisième partie examine le processus de mémorisation adopté par la fille, qui nie l'écriture. Elle choisit de se rappeler son père en recourant à plusieurs moyens pour évoquer les souvenirs : la contemplation des objets et des traces de son père, ses sens, ou encore l'incorporation ou l'identification au disparu.

Ses facultés d'évocation des souvenirs lui permettent de raviver constamment la présence de son père disparu et de se réjouir des moments passés avec lui mais aussi des sentiments qu'il éprouvait à son égard. Ainsi, le deuil reprend un nouveau sens grâce à la mémoire sensorielle qui transforme la mort en une régénération et le chagrin en une jouissance de retrouvailles.

Mots clés: deuil, mémoire, consolation.

La mort, j'entends la mort naturelle laprès une longue vie de travail et d'amour/ n'est pas une limite, une négation de la vie/ elle donne au contraire à la vie sa dimension/la plus haute, cette dimension spécifiquement/ humaine : la transcendance ${ }^{2}$ Roger Garaudy

\section{Introduction :}

Perdre une personne chère est l'ultime souffrance que peut vivre une famille, tant le lien qui unit ses membres est puissant, unique et inestimable. L'absence est cruelle et le manque infini, surtout s'il s'agit d'un adorable père de famille. Son entourage éprouve alors un sentiment de mélancolie et d'angoisse lorsqu'il entre en période de deuil.

Le professeur Marc-Louis Bourgeois, médecin neuropsychiatre et docteur en psychologie, définit le deuil comme une réaction

(Le deuil dans Muettes de Yasmine Ghata ...) Dr. Aliaa Abdel Wahed 
habituelle lorsqu'une personne est confrontée à la perte d'un être cher.

Ainsi que Marie-Frédérique Bacqué ${ }^{3}$ (2007), définit la période de deuil comme un «processus psychique lent et douloureux grâce auquel le sujet parvient progressivement à se détacher d'un être cher qui est mort ${ }^{4}$, Ainsi, l'abattement et l'affliction s'emparent des survivants.

Dans le roman de Yasmine Ghata, Muettes, le père de famille décède en laissant une épouse et une fillette âgée de 6 ans. Toutes deux doivent surmonter la période de deuil, et la vivent différemment selon leur âge et leur degré d'attachement:

Pour la mère, on souligne que «La mort d'un conjoint est reconnue comme un stress de grande intensité. Elle oblige à faire à la fois le deuil d'une personne et le deuil du couple. Le veuf ou la veuve est exposé(e) à la solitude, au manque de vie relationnelle intime. $\gg^{5}$.

Quant à l'enfant, «Quand la mort touche un enfant jeune ou un bébé à la naissance, l'interrogation sur le sens peut devenir encore plus douloureuse. L'enfant ou l'adolescent qui perd un parent, un frère ou une sœur, voit tout l'équilibre familial remis en cause. ${ }^{6}$

Tant d'écueils résultent de la perte d'un être cher pour le survivant «c'est à cette étape là que l'endeuillé a le plus besoin de soutien, de l'affection de ses proches, d'écoute aimante et chaleureuse. ${ }^{7}$

(Le deuil dans Muettes de Yasmine Ghata ...) Dr. Aliaa Abdel Wahed 1703 
Stig Dagerman ${ }^{8}$ souligne que «notre besoin de consolation est impossible à rassasier » ${ }^{9}$ on ajoute que Marie-Frédérique Bacqué souligne que «le rôle du groupe dans le deuil individuel est fondamental, ${ }^{10}$

En fait, «les mots réconfortent, permettent d'extérioriser la tristesse qui étouffe. ${ }^{11}$ «Atténuer la tristesse, c'est déjà consoler $»^{12}$ et « La consolation, c'est le langage considéré comme instrument de thérapie $»^{13}$. Une parole ou un geste de réconfort peuvent aider la personne endeuillée à entendre que perdre un être cher est une épreuve douloureuse qui a besoin de temps pour guérir.

Or, les personnages de Muettes refusent toute consolation et vivent le deuil dans le mutisme. Le manque d'apaisement entraîne alors une extrême affliction. Le deuil est vécu dans la solitude, la souffrance et la dépression, tandis que «l'accompagnement des endeuillés reste l'une des meilleures façons de resocialiser le deuil et la mort ${ }^{14}$

De surcroît, le pouvoir subversif du chagrin risque de plonger la mère et sa fille dans un état maladif : si l'on n'est pas consolé, on provoque ainsi notre propre malheur, ainsi le refoulement de la mort diminue et la réalité affligeante apparait dans les moments d'angoisse.

En conséquence, l'absence de réconfort constitue un risque durant la période de deuil qui peuvent être en proie à un effondrement dépressif puisqu'«on considère que le soutien

(Le deuil dans Muettes de Yasmine Ghata ...) Dr. Aliaa Abdel Wahed 
social de la famille et des amis a un effet direct positif sur la santé et qu'il protège l'individu des conséquences du deuil» ${ }^{15}$

Dans cette recherche, nous nous interrogeons à partir de ce point : comment parvenir à surmonter un deuil en étant inconsolé et inapaisé, à vivre des moments de bonheur durant le deuil sans ressentir l'absence de la personne aimée disparue ?

Nous tenterons de répondre à cette question en adoptant une approche psychosociale. En effet, il nous semble important de décrire les différents états vécus dans le silence par les personnages endeuillés du roman en distinguant trois axes :

- le deuil inconsolé, où nous montrerons l'état dans lequel les endeuillées se trouvent sans accompagnement ni soulagement extérieur ;

- le deuil scriptural, un moyen adopté par la mère pour surmonter cette période douloureuse ;

- et enfin, le deuil mémoriel, un processus où la fillette convoque les souvenirs des moments passés avec son père comme un dernier moyen de se remettre des souffrances liées à l'absence de ce dernier.

\section{Le deuil inconsolé}

On souligne à propos du deuil que «La période centrale du deuil est constituée chez l'enfant comme chez l'adulte par un

(Le deuil dans Muettes de Yasmine Ghata ...) Dr. Aliaa Abdel Wahed 
véritable état dépressif ${ }^{16}{ }^{6}$, Le décès du maître de la maison constitue un choc brutal pour son épouse et sa fille.

Cette dernière, qui est aussi la narratrice, met du temps à comprendre que son père ne reviendra plus. Elle ne parvient pas à imaginer son absence. Le lien entre ces deux êtres reste intense et intime, si bien que la fillette de 6 ans ressent un manque de sécurité, d'amour et de tendresse qui attise les douleurs causés par l'épreuve et les regrets liés à la rupture.

«Le décès d'un parent oblige l'enfant à se défaire de l'illusion sécurisante selon laquelle ses parents sont invulnérables et immortels. Cette prise de conscience peut être source d'une forte angoisse se traduisant par une dépendance anxieuse. $»^{17}$

En conséquence, le quotidien s'estompe pour céder la place à l'absence dramatique du géniteur. La fracture émotionnelle s'aggrave lorsque le mot «décédé » est inscrit sur les papiers officiels. La déprime assaille l'enfant, désormais en manque d'attachement paternel.

Elle garde dès lors ses yeux ouverts et écarquillés, à la recherche de son père dans toutes les pièces de la maison. Elle imagine son existence partout et nulle part, elle croit l'écouter, le voir, et a l'impression que les yeux de son père la scrutent à chaque instant.

On souligne qu'«une telle dépression est augmentée significativement lorsque la mort du parent survient avant que l'enfant n'ait atteint l'âge de onze ans ${ }^{18} \mathrm{La}$ confrontation au décès du père provoque donc un examen de la question du sens ou

(Le deuil dans Muettes de Yasmine Ghata ...) Dr. Aliaa Abdel Wahed 
des interrogations existentielles. Les larmes traduisent une volonté d'expurger la représentation de tout élément émotionnel.

L'enfant narratrice ne cesse de poser cette question «il va revenir aujourd'hui , papa ?» ${ }^{19}$ cependant, sans réponse de la part de sa mère, l'enfant se décrit : «ce jour-là, j'ai laissé mes larmes couler sans lutter,(...). » ${ }^{20}$

Les larmes traduisent une volonté d'expurger la représentation de tout élément émotionnel. Mélancolique, elle décrit que les deux mots «larmes» «et «drame» résument « la singularité des premières années de ma vie $»^{21}$.

La fillette vit le deuil dans un chagrin excessif et interminable à travers lequel «la personne endeuillée sent que les mauvais objets dominent, que son monde intérieur est en danger et que son Moi est attaqué par les pulsions destructrices. $»^{22}$

Quant à la mère, le deuil marque pour elle la fin d'une relation conjugale, créant une sensation de perte et de frustration. Les bons moments étaient comblés par l'époux, mais une fois celui-ci mort, le délire du deuil encombre la veuve, qui prend alors conscience du caractère irrémédiable de la perte. Les larmes et la dépression se succèdent sans relâche. Elle répète des mots incompréhensibles à travers ses sanglots, mais ce sont des mots ambigus et décousus.

A travers ses pleurs, elle répète des mots incompréhensibles. elle «n'a jamais pu se résoudre à dire la réalité de manière objective.» ${ }^{23}$ mais ce sont des mots masqués, ambigus et décousus.

(Le deuil dans Muettes de Yasmine Ghata ...) Dr. Aliaa Abdel Wahed 
La psychologue Joyce Chagnon a souligné que les différentes réactions $\mathrm{du}$ chagrin sont des états de douleur subjectifs et inexprimés qui accompagnent la perte de l'époux. Le partenaire survivant se sent alors incomplet, perturbé et incapable de se reconstruire

La mère subit plusieurs manifestations qui montrent son égarement. On souligne qu'en deuil: «plusieurs facettes de désorganisations peuvent apparaitre: différents degrés de distraction, une mauvaise concentration, une confusion et un manque de clarté . ${ }^{24}$

Le trouble s'empare d'elle alors que la narratrice «la voyais se débattre dans une confusion de mots. ${ }^{25}$, elle perd tout repère, et est incapable d'organiser ses pensées. Elle cache une détresse silencieuse, une affliction infinie qu'il ne lui est pas facile d'accueillir. En conséquence survient un certain ralentissement cognitif engendré par une mélancolie bien particulière.

L'intense alanguissement pour la personne défunte laisse un certain ralentissement cognitif qui se manifeste car la génitrice ne se concentre que sur le sujet de la perte: «les personnes endeuillées peuvent présenter une pensée ralentie et une mémoire appauvrie ${ }^{26}$

La narratrice assiste à la régression mentale de sa mère : «elle se préparait sans finir de s'habiller, me consolait sans finir ses phrases et composait des numéros erronés ${ }^{27}$, alors qu'elle s'incline affolée et étonnée de son malheur.

(Le deuil dans Muettes de Yasmine Ghata ...) Dr. Aliaa Abdel Wahed 
L'enfant rajoute que "ma mère relevait la tête et murmurait la phrase du bout des lèvres, les mots dissonants disparaissaient après un court passage dans l'air. ${ }^{28}$, des pensées intrusives et une détresse de séparation qui provoquent une confusion mentale.

La psychologue Emmanuelle $\operatorname{Zech}^{29}$ affirme qu'il existe un conflit entre le monde sans le défunt et le moi qui lui reste attaché, ce qui laisse libre cours aux affects pénibles. La mère se laisse emporter par l'angoisse, et sombre dans une profonde douleur qui se traduit par des gestes absurdes :

la narratrice décrit que « ses gestes théâtraux indiquaient à la fois sa colère, son impuissance et sa détresse. $»^{30}$ son trouble se manifeste lorsqu'« elle se préoccupe à enfiler ses bas à maquiller ses cils à enchaîner ses gestes sans ordre logique $»^{31}$

La fillette dépeint également l'attitude de sa mère, surprise par le choc émotionnel du décès: «ma mère avait perdu toute cohérence, au point mort, incapable de finir ses gestes. ${ }^{32}$ Seule, l'enfant contemple les gestes de sa mère et ne parvient plus à l'aider.

Devenue orpheline de père, elle est triste face aux crises de douleur mentale de sa mère. Car comme souligne E. Zech «il paraît évident que la perte d'un conjoint va également créer une perte de soutien émotionnel. ${ }^{33}$ Le manque d'énergie rend toute entreprise difficile ; par conséquent, le sommeil et la concentration sont perturbés.

le discours de la narratrice reste à ce titre significateur : "Pas une trace de sommeil dans sa voix, deux raclements de gorge

(Le deuil dans Muettes de Yasmine Ghata ...) Dr. Aliaa Abdel Wahed 
suffisaient à en ôter toute la brume du rêve. » ${ }^{34}$ il s'agit d'un psychisme blessé, accompagné par l'insomnie de la mère décrite par sa fille :

«Elle ne se nourrissait plus que de sommeil, trois prises par jour d'un repos lourd et accablant. » ${ }^{35}$

Dr Christophe Fauré définit cet état comme une «cicatrisation psychique ${ }^{36}$ accompagnée par l'insomnie de la mère, qui décrit sa fille. Le processus de deuil déclenche une sensation de perte générale, aux niveaux mental, spatial, social, dont l'encombrement mène à la digression. :

«ma mère était de la lave en fusion, un chaos perpétuel. » ${ }^{37}$, Cet état est affirmé par Joyce Chagnon au niveau émotionnel que « la perte d'un conjoint est l'expérience la plus stressante que peut vivre un individu $»^{38}$.

En effet, le deuil peut avoir un impact négatif sur le psychisme, induisant chez la personne endeuillée des comportements qui la mènent à rechercher l'être perdu. Les attitudes de la mère sont révélatrices à cet égard.

«Elle avait respiré chaque carton l'un après l'autre à l'affût de son odeur, et avait retrouvé celle de sa peau, parfois celle de sa sueur. ${ }^{39}$

A la limite de la folie : «elle chuchotait dans l'obscurité, tentant de communiquer avec l'esprit de mon père ${ }^{40}$ Elle vit dans la tristesse, et la colère, à travers une litanie des chagrins prononcée

(Le deuil dans Muettes de Yasmine Ghata ...) Dr. Aliaa Abdel Wahed 
en silence par le moi en mal de l'autre, état où elle est impuissante face à l'impossibilité de trouver le conjoint absent.

Cette période de deuil demeure cette désolation soulignée par Julia Kristeva, «qui serait ainsi le négatif de l'omnipotence, l'indice premier et primaire que l'autre m'échappe, mais que le moi, cependant, ne s'accepte pas abandonner. ${ }^{41}$

Elle se rebelle contre la réalité, et son désir intense de retrouver son mari décédé peut parfois provoquer des hallucinations visuelles. ${ }^{42}$ «Au réveil, ma mère avait oublié sa disparition et le cherchait dans la pièce voisine, persuadée que la mort n'était pas mort. ${ }^{43}$

Des crises de tristesse envahissent la mère. : « Ma mère se réveillait la nuit, en proie à des flambées anxieuses, des affolements qu'elle retenait jusqu'au matin. ${ }^{44}$, De plus, le monde devient étroit à ses yeux : «ses problèmes lui paraissaient alors insolubles et permanents. ${ }^{45}$ Ainsi, «la nuit entretenait ce fantasme du pire. ${ }^{46}$

Devenue une autre, la mère est décrite par sa fille à ses mots : «sa tête lourde lui imposait un court somme toujours interrompu par la sonnerie de téléphone. »22 les voix deviennent pour elle «des bombardements sonores », et les talons de sa mère sur le parquet lui semblent comme des «martèlements pressés. » ${ }^{47}$

La génitrice vit de terribles moments de souffrance, La narratrice poursuit sa présentation : «Ma mère s'était accroupie au pied du mur, sanglotant dans le creux de ses genoux. Elle répétait

(Le deuil dans Muettes de Yasmine Ghata ...) Dr. Aliaa Abdel Wahed 
le prénom de mon père, mêlant larme et salive d'une même eau. ${ }^{48}$

Et même son corps montre des signes d'épuisements, d' "une perte significative (...) peuvent comprendre des sentiments de tristesse intense, des ruminations à propos de la perte, une insomnie, une perte d'appétit et une perte de poids, ${ }^{49}$

L'enfant décrit à ses mots : «ma mère avait maigri, beaucoup maigri. Elle se traînait sans fouler le sol, à petits pas. Je la voyais rétrécir chaque jour, s'amoindrir. ${ }^{50}$ «ma mère avait la bouche sèche et le bout des doigts racorni. » ${ }^{51}$ L'enfant rajoute que «ma mère relevait la tête et murmurait la phrase du bout des lèvres, les mots dissonants disparaissaient après un court passage dans l'air. » 52

La perte de son époux déclenche l'abandon de soi, car son décès habite sa veuve et structure d'ores et déjà sa vie. La narratrice ajoute :« Ma mère, elle, continuait à traîner sa vie en va-et-vient ou déambulations rituelles. Le rythme de ses pas détachait les mots, c'étaient des lamentations incompréhensibles tant la souffrance déformait son souffle. $»^{53}$

Cependant, le deuil inconsolé permet à la mère et à sa fille de se tenir loin de tout contact social. Repliées sur elles-mêmes, murées dans le silence, elles fuient les sonneries du téléphone, décrites comme des bruits extrêmement ennuyeux ou des «vacarmes au comble de leur ennui $\gg^{54}$

(Le deuil dans Muettes de Yasmine Ghata ...) Dr. Aliaa Abdel Wahed 
Lorsque le téléphone sonne, la mère répond, absente d'ellemême. Les appels incessants la laissent indifférente. La peine ressentie les amène toutes deux à ne plus percevoir les comportements d'aide et de soutien. Leurs souffrances sont cachées du regard de la société, alors qu'elles sont profondément douloureuses.

Ce monde devenu étroit limite leur vie et leur conscience au point qu'elles ne trouvent que la claustration. Emmanuelle Zech souligne que «par périodes succédant aux moments d'intrusions mentales, les personnes endeuillées peuvent éviter les pensées liées au décès ou encore éviter les endroits qui rappellent le décès. » ${ }^{55}$

L'évitement est une réaction issue d'une détresse intense où les endeuillées refusent toute compassion. La fille décrit sa mère à ce propos :« Elle se disait agressée par les condoléances, la colère lui avait fait dire un jour qu'il n'était plus mort. Revenu après une longue absence. ${ }^{56}$, le deuil inconsolé est manifesté par des attitudes manifestes ainsi :

Pour la mère : «Dès lors, elle refusa les visites amicales, les regards réconfortants, les paroles apaisantes, les apitoiements et les soupirs impuissants. »30 alors que la fille s'exprime : «je n'avais besoin de personne ${ }^{57}$ Les personnages passent le deuil dans le mutisme en affirmant qu'« il n'y avait pas de mots pour dire la souffrance $\gg^{58}$

(Le deuil dans Muettes de Yasmine Ghata ...) Dr. Aliaa Abdel Wahed 
Par conséquent, la maison est envahie par le silence triste, les regards sont recueillis dans le désarroi de la veuve et le déni du deuil pour l'enfant qui explique : «notre appartement était devenu la scène d'une tragédie, et la culpabilité, notre pain quotidien. » ${ }^{59}$, Le monde est dépourvu de réactions, de signifiance, il est muet et silencieux: «la maison était devenue un lieu de passage, de regards recueillis et de silences tristes. ${ }^{60}$ «ma mère s'exilait dans sa chambre ${ }^{61}$

Le deuil inconsolé rend leur domicile dénué d'esprit, la narratrice déclare que «ce couloir n'aurait plus de sens. ${ }^{62}$ tandis que l'espace reste à leurs yeux un égarement où les fers forgés des balcons se transforment en « plusieurs labyrinthes s'y croisaient et s'y pénétraient sans que je puisse en deviner le départ ni la sortie. ${ }^{63}$

Toutes deux se débattent sur le seuil de l'abîme. À défaut de consolation ou de soutien, la mère et sa fille ne parviennent ni à surmonter leurs souffrances, ni à reprendre le contrôle d'ellesmêmes. Elles risquent de tomber dans un deuil infini ou pathologique.

«Dans le deuil le monde est devenu pauvre et vide, dans la mélancolie c'est le moi lui-même. ${ }^{64}$. ce qui favorise la survenue de différents formes d'atteintes sur la santé tant physique que psychique.

L'abattement silencieux des deux personnages est associé à la mort et au chagrin funèbre, un état rapporté par la parole de la

(Le deuil dans Muettes de Yasmine Ghata ...) Dr. Aliaa Abdel Wahed 
fille : «nous vivions muettes, les mots n'avaient pas leur place ici $»^{65}$, alors que la parole de la mère manifeste cet état « Dans ses larmes, elle disait des choses qu'elle n'avait cessé de taire ces huit derniers mois. Des mots terre à terre sans poésie et sans imaginaire. ${ }^{66}$

C'est le moment où toutes deux ont davantage besoin d'une consolation faisant appel au courage et à la fermeté d'âme. Or, les relations sociales apportent du réconfort, et permettent d'exprimer les sentiments.

«Le deuil peut entraîner un individu vers un état psychologique potentiellement dangereux, si l'émotion n'est pas limitée et prise en charge par la collectivité. » ${ }^{67}$ La narratrice explique clairement leur état de deuil inconsolé, étouffé dans le mutisme et l'absence de l'être cher. :

«Ma mère vit ces huit derniers mois sans mon père matérialisés dans ce sombre décor. J'ai dessiné un graffiti sur la table, un cœur sans doute, à l'aide de mon index. Elle regarda le ciel comme pour lui parler. Des murmures plein la bouche qu'elle étouffa de sa main. Elle essuya sa joue, elle dessina deux autres cœurs sur la table à proximité du mien. (...). Sans un mot, je traçai une ligne séparatrice qui lui signifiait que l'un d'eux était passé de l'autre côté. » ${ }^{68}$

Par conséquent, le deuil ne peut être englouti ni par le passage du temps ni par l'oubli. En effet, l'expérience de la perte donne lieu à

(Le deuil dans Muettes de Yasmine Ghata ...) Dr. Aliaa Abdel Wahed 
la recherche inlassable d'un substitut au risque que la douleur ressurgisse plus ou moins violemment ultérieurement.

«Cette perte et cette séparation vont entraîner une souffrance et un processus biopsychosocial de changement qui exige une adaptation. » ${ }^{69}$ Dès lors ,un travail de deuil reste indispensable où les endeuillées décident de s'autoconsoler par «une démarche active qui permet de canaliser la douleur et d'aller vers la guérison

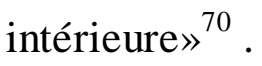

\section{Le deuil scriptural}

«Le deuil conduit jusqu'au bout produit une maturation de la personne qui ne sera jamais «plus comme avant» car elle peut transformer en enrichissement affectif et intellectuel les épreuves passées ${ }^{71}$

De ce point de vue, la mère s'oriente vers une auto consolation par l'écriture qui représente, à ses yeux, la seule échappatoire à la réalité. L'acte d'écrire peut être considéré comme le premier geste menant à la recherche d'une vie qui ne dépend pas des autres mais à travers laquelle il est possible de s'oublier dans un monde créé par soi. Par la suite, elle a l'impression de «prendre l'univers en main ${ }^{72}$

En fait, la réalité est reportée à l'arrière-plan au profit de l'écriture. La narratrice dévoile le penchant de sa mère pour sa machine à écrire. : «c'est la machine à écrire de ma mère ; indifférente à son deuil, qui sortit ma mère de cet abîme. ${ }^{73}$, Freud souligne également que signifie cette période d'écriture

(Le deuil dans Muettes de Yasmine Ghata ...) Dr. Aliaa Abdel Wahed 
dans le deuil : «ménager un espace de «non-deuil» à l'intérieur du deuil. ${ }^{74}$

Le chagrin issu du deuil amène ainsi à une évolution créatrice. ${ }^{75}$ un chemin «à sonder l'imaginaire ${ }^{76}$ où «le monde réel fut d'emblée relégué sur les bas-côtés $»^{77}$ décrit la narratrice. Les mots permettent de transcrire le deuil par une technique rhétorique, qui console l'affligée grâce à des scènes fantaisistes, capables d'apaiser sa douleur et de lui fournir une assistance.

«Écrire la purgeait de ses culpabilités inconscientes, celles qui nous consument bavant le labeur, et qui nous quittent au dernier paragraphe gagné sur la page blanche. Après ces deux heures de travail quotidien, elle était une autre, vidée comme à marée basse;(...). $\gg^{78}$

La mère projette ainsi sur le texte l'expérience d'un vécu individuel authentique et lisible afin que l'écriture devienne une voie purgatoire pour dépasser l'épreuve.

« Cette transformation incessante de la réalité la distinguait toujours comme seul narratrice possible, la seule à donner du souffle aux personnages, à décrire le décor. ${ }^{79}$

Les mots de la narratrice demeurent à ce propos significateurs : «Ma mère n'a jamais pu se résoudre à dire la réalité de manière objective, habituée à inventer des histoires, elle tenait de corriger l'inachèvement des choses $»{ }^{80}$

L'écriture du deuil apparaît d'abord comme une manière de contrer le mutisme résultant du deuil en intégrant dans le récit

(Le deuil dans Muettes de Yasmine Ghata ...) Dr. Aliaa Abdel Wahed 
l'expérience de cette perte. la fille décrit que « la vie était selon elle un long roman sans titre, sans alinéas et sans ponctuation, il fallait trouver son souffle au détour d'un mot. ${ }^{81}$ l'écriture reste un moyen de distraction :

«À tout moment de la journée, elle consignait sur une feuille les bonnes idées qui lui traversaient l'esprit. Ce pouvait être la nuit, après un songe loufoque, ou juste après la sieste. Le sommeil lui inspirait des images fertiles que la conscience ne censure pas. ${ }^{82}$ L'écriture apparaît d'abord comme une manière de contrer le mutisme résultant du deuil en intégrant dans le récit l'expérience de cette perte. Écrire reste un moyen de distraction, et lui permet, à travers les lettres et les mots, de présenter le monde endeuillé sans larmes, ainsi que des personnages légendaires issus de l'audelà.

La mère manie un nouveau monde dont le contenu est une transcription de la réalité à travers les images où « elle imagina la vie d'un peuple méconnu dont on ignorait tout : les morts et leur vie souterraine. $»^{83}$

Elle oublie son chagrin lors de ses recherches par l'écriture.« (...), ma mère était perdue dans ses pensées, préoccupée par l'amorce d'une phrase ou la fin d'un paragraphe. ${ }^{84}$

Elle transforme la douleur de l'absence en une cuvre littéraire qui compense la disparition de son époux en le poursuivant psychiquement à travers un monde lugubre empli de cimetières et

(Le deuil dans Muettes de Yasmine Ghata ...) Dr. Aliaa Abdel Wahed 
de morts qui se parlent. De cette façon, le deuil devient une période d'une singularité littéraire irréductible.

la narratrice explique le monde créé par sa mère : «(..), ma mère était passé de l'autre côté de la vie, dans cet espace où l'imagination est abri et refuge. La mort de mon père n'avait fait qu'accentuer ce besoin viscéral de tailler la réalité, dégrossir la matière et n'en retenir qu'une substance polie par les mots, ciselée par les lettres. ${ }^{85}$

Ainsi, le chagrin débouche sur un processus transformatif et intersubjectif de la veuve, qui parvient à créer une nouvelle façon de vivre son angoisse avec le monde de son défunt époux, celui des morts, et à assurer de cette façon une liaison continue avec eux à travers le texte.

«L'épreuve de la narration, en inscrivant l'insaisissable expérience de la perte dans le temps par une opération de mise en intrigue, permettrait au sujet de l'intégrer dans la continuité de son histoire personnelle, de l'inscrire dans une totalité racontable. À travers ce processus, la personne parviendrait à donner du sens à sa perte et à reconstruire son identité altérée par le deuil. ${ }^{86}$

Le récit devient dès lors « un lieu de symbolisation de la perte, favorisant la mise en œuvre d'un travail de deuil et l'attachement à un nouvel objet libidinal. ${ }^{87}$ où la rupture et l'absence réelle font l'objet d'une configuration imaginaire dans l'écriture.

la narratrice surenchérit la signification de l'œuvre de sa mère vis-à-vis du deuil : «L'analyse des faits était le point de départ

(Le deuil dans Muettes de Yasmine Ghata ...) Dr. Aliaa Abdel Wahed 
d'histoires où le mythe auréolait des acteurs inconscients de leurs propres rôles. Le monde réel était transformé par des mots sans modération et sans mesure. ${ }^{88}$

Les critiques décrivent le travail du deuil comme étant « un long processus scriptural et poétique, où les voix des autres - celles des intertextes et celles de ses spectres — se mêlent à la sienne ». ${ }^{89}$ Ce sont « Des mots enchevêtrés qui dessinaient les contours d'une silhouette, la densité d'un corps disparu. Mille astuces et mille images. ${ }^{90}$,surenchérit la narratrice

Cette polyphonie énonciative fait partie intégrante de l'écriture de la mère, un acte qui lui est absolument nécessaire pour modifier l'aspect de son désespoir et pour parvenir à tempérer les manifestations intenses de la détresse. Ce sont «Quelques mois de contes et légendes alimentés par des allégories fabuleuses, des facéties burlesques pour maquiller son absence. ${ }^{91}$

Quand elle continue à écrire les voix des disparus, toujours en quête d'un apaisement et d'une consolation, elle rejoint une communauté de personnages qui lui permet de ne jamais se sentir seule. C'est ce détour par la fiction qui rend l'épreuve du deuil infiniment singulière. La veuve s'évertue à faire parler les personnes disparues malgré le silence de la mort comme le décrit le critique Dominique Rabaté :

«Donnée à lire et à voir peut faire écho à l'expérience même de la littérature qui ne cesse de lier présence et absence à travers l'aventure du langage, entretenant ainsi «l'incessant dialogue

(Le deuil dans Muettes de Yasmine Ghata ...) Dr. Aliaa Abdel Wahed 
entre les vivants et les morts $»^{92}$. L'écrivaine s'évertue à les faire parler malgré le silence de la mort.

la grande psychologue Julia Kristeva précise toutefois que la création esthétique peut aussi constituer une façon de se complaire dans la souffrance, de jouir de la contemplation de l'absent transcrit par le langage littéraire, nécessaire pour vaincre la mélancolie issue de cette perte initiale, la dissout dès lors qu'il la représente ${ }^{93}$

En fait, par le biais des métaphores traditionnelles, l'affliction devient une métonymie pour exprimer le manque et les non-dits en mettant en scène le deuil de façon topique. La narratrice souligne à ce propos :

«La disparition de mon père avait inspiré ses plus beaux poèmes, mais la vie tout entière était devenue à ses yeux une strophe de poème où le mot «mort» est sans cesse paraphrasé. Exercices et figures traduisaient son refus ${ }^{94}$

Les techniques et les raisonnements ordinaires ne parviennent pas à la consoler de cette disparition. Seul l'espoir de rendre hommage par l'écriture à son époux disparu semble pouvoir atténuer sa douleur par le biais du langage fleuri des discours.

«Le rituel du café bu par petites gorgées lui inspirait ses plus belles pages. Elle retournait sa tasse pour lire les méandres du marc avec le même recueillement que ses pages corrigées. Le bestiaire fabuleux dessiné sur la porcelaine était aussi romanesque que sa prose, les poules couvaient un manuscrit en préparation, le 
coq chantait son triomphe et le scorpion nichait au sommet de la montagne. ${ }^{95}$

En effet, elle s'inspire des signes qu'elle voit dans le marc de café, au fond de sa tasse pour écrire et explorer la part inventive de son caractère. Ainsi, elle oublie sa tristesse et se protège de sa propre destruction grâce aux mots qui mettent à l'épreuve son état et qui, par la suite, permettent une plus grande liberté littéraire.

Le recours urgent à l'écriture transforme le processus de deuil en une œuvre littéraire. A ses yeux, « La fiction était toutepuissante ${ }^{96}$ l'écrivaine - mère était « une créature en transition entre deux espaces. ${ }^{97}$ ceux de l'imaginaire et de la réalité.

L'écriture devient la parole des défunts, et une figuration textuelle des disparus. Bacqué, M.F. a souligné que «Le deuil est un processus. C'est une succession de mouvements psychiques qui vont remanier totalement la vie d'un individu ${ }^{98}$ La narratrice surenchérit à ce propos :

«Ma mère ne quittait plus sa machine à écrire, sans doute pensait-elle établir un lien avec l'au-delà en martelant les touches comme pour ouvrir une serrure. Elle voulait forcer ce barrage interdit au moyen des mots. $»^{99}$

Cependant, l'écriture incessante de la mère fait voir « la vie comme un prolongement naturel de sa prose, les mêmes métaphores, les mêmes figures de style transposées dans notre quotidien. ${ }^{100}$, explique la narratrice. Le monde lugubre de la 
réalité se prolonge par l'écriture en un univers sinistre et imaginaire.

La mère s'immerge dans un monde invraisemblable, qui tend à manipuler la réalité. : « (...) elle tentait avec fièvre d'établir un lien avec la réalité, celle des autres, d'un monde littéraire à la fois tangible et faux.» ${ }^{101}$, où «l'affabulation emprunte les voies détournées de la fiction et qu'elle engendre d'autres signes, d'autres mots ou d'autres images, ${ }^{102}$

En effet, l'écriture est toujours motivée par un sentiment de perte, lorsqu'elle ne parvient plus à redonner vie à l'être disparu par le truchement du texte, ni à avoir une emprise sur la mort avec les mots :

«Ecrire pour retrouver mon père. Elle se jugea impuissante, les mots n'arrivaient pas à destination, immobilisés sur un seuil que personne n'avait franchi auparavant. Le rythme ascendant de ses phrases harcelait le néant et l'absence. » ${ }^{103}$

L'écriture de la mère veut celle du deuil, de l'anéantissement, puisqu'elle ne parvient pas à dresser un portrait fidèle de son époux défunt, les mots dénaturant la réalité. Par conséquent, le deuil scriptural ne fait que dédoubler la sensation de la perte par la création d'un univers mythique et lugubre empli de cimetières. La progénitrice décrit l'écriture de sa mère ainsi :

«les morts lui inspiraient des phrases courtes, sans paraphrases et sans métaphores. Elle élaguait, enlevait l'écorce des mots et débitait leur chair, écho à la longue dégradation des corps. » ${ }^{104}$

(Le deuil dans Muettes de Yasmine Ghata ...) Dr. Aliaa Abdel Wahed 
Par ailleurs, le monde de l'écrivaine est silencieux, habité par des défunts muets et énigmatiques, auxquels elle tente de donner une voix. À force d'écriture, il n'y a plus de place pour le vocabulaire relatif à l'absence et au vide.

«De cette cendre naissaient des phrases alitées, un texte sans respiration, sans ventilation, où les espaces se meurent et les syllabes agonisent. ${ }^{105}$

La mère s'efforce de mettre en mots la mort indicible, mais dès qu'elle tente d'insérer ce sujet dans le langage, celui-ci la trahit, et l'écriture devient une symbolisation du néant.

La fille décrit ainsi le style d'écriture de sa mère à ses mots : «Ma mère reportait les caractères indéchiffrables couchés sur le papier. ${ }^{106}$ «Des mots obscurs, sans vie, des formules pétries d'une glaise fuyante et imprégnée des défunts. ${ }^{107}$

Ce sont des textes écœurants, des mots macabres et morbides. Désinvesti de sens, le langage du deuil ne détient plus le pouvoir de produire même l'insignifiance de la mort dans sa complexité et son essence :

« la dimension du langage s'avère insuffisante, le sujet retrouve dans la situation, sans issue, du désarroi qui débouche sur l'inaction et la mort. ${ }^{108}$

Autant les lettres modifient le réel et le rendent fictif, autant la mort crée un espace livresque clos. L'écriture inlassable de la mère est confinée dans cette zone ombreuse et touffue des 
cimetières et de l'absence du langage, où ne se font entendre que les appels des souterrains et des défunts au lointain:

«L'enveloppe kraft avait englouti les pages, retenant désormais l'imaginaire dans un espace clos. ${ }^{109}$

alors que l'écrivaine est étouffée par l'insuffisance des mots et la clôture du livre. En conséquence, la douleur n'est ni dissoute ni apaisée, et le chagrin, comme aux premiers jours du deuil, est décrit au fil des notes.

«Elle s'évertuait à trouver les bons mots, mais, bien trop souvent, ces belles images et ces belles tournures puisaient leur poésie dans l'absence totale de vraisemblable. ${ }^{110}$.

Il s'agit d'une écriture endeuillée, car chaque mot, chaque scène correspond à une justification de la mort et de l'absence. La narratrice décrit cet état à ses mots :

«C'est cette machine à écrire (...) qui officialisa la mort de mon père. ${ }^{111}$ L'écriture devient alors une contrepartie de la désolation.

Par ailleurs, l'écriture détache la veuve du quotidien. L'acte littéraire la mène en dehors de la vraie vie, dont elle s'éloigne, car elle ne parvient pas à l'affronter. Le discours de l'enfant reste à ce propos révélateur :

«Migrante passive, elle avait fui la vie à sa façon, m'avait fuie. Une présence trompeuse car son esprit vagabondait. Elle était là et ailleurs, concrète et manquante. ${ }^{112}$

(Le deuil dans Muettes de Yasmine Ghata ...) Dr. Aliaa Abdel Wahed 
Cela tient au fait que l'écriture de son deuil repose sur la construction d'un monde imaginaire et sur un lent processus de sédimentation qui réagit à contre-courant de la vie quotidienne.

La narratrice surajoute que « Son obstination à raturer, recommencer la même phrase, la même page, l'avait coupée de nous. A mes yeux, son écriture quasi automatique, enterrait mon père chaque jour un peu plus profondément. ${ }^{113}$

Le discours de la narratrice demeure significatif à ce propos : «Ses yeux n'étaient plus les mêmes quand elle était capturée par une fiction naissante. L'inspiration lui dictait de folles images qui contaminaient aussi soudainement notre voie commune. ${ }^{114}$

D'ailleurs, la vie quotidienne lui échappe en raison d'une démystification du réel par l'écriture. Par la suite, son présent perd son épaisseur, son sens, et se réduit à sa dimension la plus imaginaire et la plus incohérente.

À ce moment-là, la veuve ne surmonte pas son deuil et ne vit pas non plus son quotidien. En outre, l'écriture demeure une phase temporaire au terme de laquelle elle achève son œuvre et affronte la réalité à son comble.

La narratrice la décrit comme «Fragile laps de temps qui lui imposait la réalité en face ${ }^{115}$, La fiction la plonge dans les mots funèbres, une interruption qui ne dure que quelques semaines.

L'évanescence de l'écriture peut être due à l'écoulement du temps: «La disparition de mon père avait broyé le lyrisme de ma mère,(...).» ${ }^{116}$ En fait, ce basculement entre l'imaginaire et la 
fiction permet, avec le temps, de mettre fin à l'imagination qui se tarit au fil des semaines.

De même, la fugacité de l'écriture est attestée par la précarité des papiers qui peuvent être détruits par plusieurs incidents. Ils s'envolent occasionnellement, emportés par le mistral:

«Je la laissais sur la terrasse, à ses feuilles griffonnées, à ses vertiges et étourdissements qu'elle calmait par des douches fraîches à intervalles réguliers. Ses lignes escaladaient les angles de la page, grimpaient et dégringolaient avant de s'échouer, bousculés par le mistral. ${ }^{117}$

Dans le même registre, l'incendie qui dévaste les collines en été balaie les traces de son écriture en une nuit: «Le feu avait dévasté des collines de pins parasols en une nuit, et il avait balayé sa mémoire ${ }^{118}$

La panne de la machine à écrire interrompt également le processus durant quelques semaines. Ainsi, l'écrivaine subit plusieurs ruptures successives dans son acte d'écriture qui lui permet de faire face à la dure réalité de l'absence de son conjoint qu'elle essaie d'oublier :

«En proie au réel, elle ne semblait plus confisquée par autre chose. ${ }^{119}$ Dès lors, le deuil scriptural s'éloigne de la fixité, de la permanence et de la visibilité.

De surcroît, l'écriture mène la mère à la limite de l'errance. «(...), qu'écrire lui avait fait perdre toute raison, les mots la menaient au-delà de l'évidence. ${ }^{120}$ Elle vagabonde dans le 
labyrinthe des mots, où elle ne ressent pas son propre monde réel mais celui de son défunt mari dans un univers falsifié et funéraire : «Une parole qui crée un univers imaginaire et permet de reconstruire l'univers symbolique disloqué. une parole qui n'est cependant pas sans danger car elle engage le sujet dans un monde imaginaire où il peut définitivement se perdre. ${ }^{121}$

En outre, les images du deuil la submergent. Bien qu'elle tisse un monde dont elle s'accommode par le biais de l'écriture, les mots ne compensent pas l'absence de l'être cher et ne la laissent pas jouir des souvenirs. Au contraire, ils transcrivent le chagrin. Le discours de la narratrice demeure à ce titre significateur :

« les écrivains sont démunis face au néant, car il n’y a pas de mots pour le décrire. Les écrivains ne se mentent pas, ils fabriquent de simples arrangements dont ils s'accommodent. ${ }^{122}$

L'image de la mort reste une conjuration de la disparition définitive où elle fait appel à la descente au royaume des disparus. L'œuvre n'étreint que l'absence des personnages présentés sur fond de tentures funèbres.

L'écriture devient, en conséquence, un tombeau et une expérience poétique de désœuvrement qui traduit la disparition. Le deuil est ainsi dédoublé par l'écriture, qui tue par ses mots lugubres la voix du disparu, et qui plonge ainsi l'écrivaine dans un deuil continu.

En fait, «la littérature autorise cependant au survivant de pactiser avec le deuil pourtant inconsolable du disparu dont il 
préserve et salue la mémoire — qui lui, offrant là sa promesse d'éternité. ${ }^{123}$

\section{le deuil mémoriel}

Sigmund Freud observe que l'homme est incapable d'accepter la perte. L'inconscient ne renonce jamais au lien d'attachement, sauf s'il entretient ce lien instinctif par d'autres types de manifestations pulsionnelles du deuil. ${ }^{124}$

Ainsi, la réaction de la narratrice durant son deuil diffère de celle de sa mère. Elle décrit son état ainsi:

«Mourir n'empêche pas un père de revenir à la maison. Mourir est un acte comme un autre. Disparition totale qui l'empêcherait de dormir, de parler et de manger, tout ça n'avait aucun sens dans mon esprit. ${ }^{125}$

Selon le point de vue de la narratrice, la durée du choc de la perte est raccourcie, alors que le chaos de la souffrance est remplacé par l'indifférence : après tant d'émotions et de larmes, elle ne ressent plus son deuil:

«je suis sortie de moi-même ce jour-là, étrangère au chaos, comme si tout ça ne me concernait pas. $»^{126}$

Détachée de son monde, elle recourt au silence en évitant les voix qui l'entourent et qui la distraient, afin de réfléchir sur l'état présent. Son discours reste à ce titre révélateur :

«Je fuyais les ondes endiablées du téléphone ou les crissements du stylo sur le papier. ${ }^{127}$ elle se retire dans sa chambre loin des «pulsations vitales émis du salon » ${ }^{128}$

(Le deuil dans Muettes de Yasmine Ghata ...) Dr. Aliaa Abdel Wahed 
Les cliquetis ennuyeux de la machine à écrire et les sonneries incessantes du téléphone sont des perturbations mentales qui bousculent ses réflexions. À ses yeux, le projet d'écriture de sa mère comme palliatif ne constitue pas un travail de deuil réussi. Il crée un monde falsifié et abstrait. Les personnages ne font pas écho aux sentiments de souffrance. En outre, les livres sont des invitations à la fuite vers un espace éphémère et sombre. La narratrice surajoute :

«Je rejetais les livres, les évinçais de ma vie comme la peste. Lire, c'était abdiquer, c'était mourir. J'agonisais à chaque page, repoussant l'idée de me retrouver dans ce mensonge écrit. » ${ }^{129}$

D'ailleurs, la machine à écrire de sa mère constitue un rempart entre son père et elle, qui représentent respectivement la mort et la vie, alors que l'écriture recherche constamment le deuil. La fille refuse ce deuil scriptural qui, à ses yeux, justifie la mort de son père qu'elle préfère garder toujours présent dans sa vie.

D’après les psychologues modernes, «Le travail de deuil est un travail de mémoire », ressaisir ce qui c'est déjà perdu. ${ }^{130}$

Par conséquent, elle parvient à sauvegarder sa présence grâce à sa mémoire, qui lui suffit en compensation. Le deuil est dès lors investi par l'évocation continue des souvenirs. Les mots de la narratrice restent à ce propos significateurs :

«Moi, en silence, sans rien lui (sa mère) en dire, j'essayais de me rappeler la voix, le visage, le rire et le parfum de mon père. ${ }^{131}$ à partir de la création d'un univers d'illusion, d'un 
espace virtuel avec son père décédé, qui lui permet de supporter la séparation sur la base d'une image ou d'un objet.

La narratrice exhibe prestigieusement ce que «Gilles Deleuze nomme «L'illusion cinématographique de la mémoire » qui est un mouvement de «reconstruction endogène du passé » à partir de l'élection d'une image ou d'un objet qui permet de voir le père sans être vu. ${ }^{132}$

Les objets deviennent donc sa source d'inspiration pour raviver les moments les plus chers et un moyen de figuration de l'image de son père. : «Moi, j’aimais le silence, le contour des choses cernées qui délaçaient les formes en moi-même. ${ }^{133}$

Ressusciter la présence du père fait partie du défi qu'elle relève contre la mort. Elle déclare : «Ce jour-là, j’ai examiné mille objets, croyant y trouver son âme. $»^{134}$

Lorsqu'elle regarde un objet, elle se souvient d'un événement vécu avec son père, permettant ainsi à ce dernier d'exister éternellement dans sa vie.

Le deuil vécu à travers la mémoire et les objets hétéroclites va dans le sens du déni de l'irrémédiable (la mort), et permet de redécouvrir les traces du défunt:

« et puis j'ai compris un jour que je n'avais plus à attendre, je disposais de mes propres ressources pour le (père) faire revenir. Les formes, les objets sont un moyen d'évocation du passé. » ${ }^{135}$

Cette stratégie visant à vivre le deuil semble un espoir de délivrance contre l'angoisse de la disparition. La conscience

(Le deuil dans Muettes de Yasmine Ghata ...) Dr. Aliaa Abdel Wahed 
réveille les réminiscences disparues à travers un processus de «subjectivation de la mort ${ }^{136}$ expression citée par le psychanalyste Jean Lacan, qui signifie ressusciter des pensées refoulées, rendre visible l'invisible afin de combler le manque de l'autre .

La mémoire et l'imagination peuvent évoquer tout ce qui est disparu et qui peut être ranimé par les objets:

«les cartons de la cave sortiraient de l'oubli quelques années plus tard. J'aurais alors remplacé l'observation des choses par le dépouillement systématique de ces archives. ${ }^{137}$, déclare la narratrice dont «l'imaginaire vient au secours du manque. ${ }^{138}$

Ainsi, l'observation de ces derniers devient le point de départ d'une nouvelle démarche créatrice. La narratrice fréquente les coins de la maison qui gardent encore les antiquités de son père, source d'inspiration et de stimulation de la mémoire. elle décrit :

«Ce long couloir dédié aux souvenirs de mon père, ses trophées de chasse rapportés du Kenya, ses pipes collectionnées avec amour et les ossements d'un buffle reconstitués au moyen de tiges en fer. ${ }^{139}$

Désormais, ces lieux deviennent des endroits de recueillement et de retrouvailles entre fille et père. Admirer les objets de collection fait également ressentir les sentiments qui ont accompagné la démarche du père, à savoir sa passion pour les antiquités.: « ses pipes collectionnées avec amour ${ }^{140}$.

(Le deuil dans Muettes de Yasmine Ghata ...) Dr. Aliaa Abdel Wahed 
Les traits de caractère du défunt sont dévoilés par la vue de sa bibliothèque, emplie de livres saints et d'objets d'art, et reflétant son érudition en matière de religion.

«ces murs, où la Bible côtoyait le Coran, où des calligraphies arabes étaient mêlées aux aquarelles chinoises ${ }^{141}$

ce qui traduit les cultures diverses du père et son érudition au sujet de la religion qui lui permettait de garder les livres saints à sa bibliothèque. «Insignes de la loge maçonnique, taste-vin, vieux diplômes et boutons de manchettes démodés cohabitaient dans les tiroirs. ${ }^{142}$.

Cette accumulation montre sa propension à collecter des objets précieux et étrangers pour les sauvegarder.

Les objets souvenirs témoignent des moments forts passés, les rendent plus accessibles, et mettent en relation étroite la fille vivante et le père décédé. Ils constituent autant de manifestations de sa présence abstraite et de vecteurs d'un lien filial qui persiste avec le temps où «l'observation tournait à l'obsession. »" ${ }^{143}$, justifie la narratrice

Dorénavant, cette obsession devient une partie de la fillette. Les souvenirs intimes se rassemblent dans une lente revue panoramique procurant un sentiment d'apaisement.

«Les fers forgés des balcons voisins fraichement repeints avaient subi le travail acharné de mon esprit. » ${ }^{144}$

Par ailleurs, les productions artisanales qui conservent la chronique du disparu constituent, dans la maison, une sphère

(Le deuil dans Muettes de Yasmine Ghata ...) Dr. Aliaa Abdel Wahed 
intime du père. Ces objets matérialisent ses gestes et donnent vie à celui qui est désormais figé dans le temps.

«Je me rappelle mon père classant et triant ce désordre hétéroclite. Les lances africaines debout en équilibre menaçaient de tomber sur sa tête, il n'oubliait jamais de serrer les vis des articulations et jointures du buffle. ${ }^{145}$

Les objets d'art lui permettent d'effectuer une décharge pulsionnelle. Ils déclenchent les gestes effectués par l'absent, mobilisent le figé au point de le présenter comme scènes vivantes et mémorielles. La description de la narratrice est révélatrice à ce discours :

« Je le voyais, accroupi à l'entrée du couloir, dépoussiérer, classer avec minutie. Sa pipe en bouche, devenue froide à la longue, ne fumait plus. ${ }^{146}$ ces révocations mémorielles détaillées incitent à la jouissance et transforme le deuil en moment d'euphorie grâce à la rencontre avec l'autre.

En outre, l'observation et la description de ces matériaux sont accompagnées par les sens de la perception, qui jouent un grand rôle dans la prolongation de l'existence du père. Les différents sens de la narratrice facilitent l'accès au père grâce aux odeurs, aux sons, à la vue et au toucher, qui contribuent tous à créer un monde réanimé et concret.

«Je touchais son tabac à rouler, les brins aux arômes doux et épicés ramenaient à mes oreilles le frémissement de ses doigts

(Le deuil dans Muettes de Yasmine Ghata ...) Dr. Aliaa Abdel Wahed 
autour de sa pipe. Petite, j'éparpillais ses volutes de fumée, les dispersais en bondissant. $\gg^{147}$

Afin de faire face aux frustrations des réalités extérieures, les différents sens de la perception, comme le toucher du tabac fumé par le père, l'odeur qui se dégage de ses brins, l'écoute du frémissement de ses doigts, aident à imaginer et ressentir sa présence.

Elle l'indique en déclarant:«des souvenirs de famille dégageant une odeur d'encens froid. ${ }^{148}$ ainsi que «J'ai ouvert un à un les disques vinyles Deutsche Grammophon, sa collection sentait la cendre tiède. ${ }^{149}$

Ainsi, les sens deviennent une passerelle vers l'autre, et le deuil cède la place à la mémoire sensorielle. ${ }^{150}$

D'ailleurs, la narratrice nie la mort et l'oubli des êtres chers. Elle évite toute source qui brise l'élan de ses évocations: "Prise de rage, comme si je battais la mort elle-même. (...). » ${ }^{151}$ L'absence du père n'a plus de place ; en revanche, il est omniprésent grâce aux souvenirs.

La narratrice explique qu' : «A bout de souffle, je me couchais sur le ventre, comme foudroyée, je jouais à la morte. Mon père me chatouillait, mes contorsions et mes rires résonnaient dans l'appartement. Tous ces souvenirs étaient des preuves tangibles de son passage sur terre. ${ }^{152}$

La mémoire revendique les chatouillements du père et déclenche le rire de la narratrice, révélant ainsi toute une communication

(Le deuil dans Muettes de Yasmine Ghata ...) Dr. Aliaa Abdel Wahed 
effective et affective entre celui qui est absent et celle qui est présente, l'au-delà et le quotidien.

«Et les souvenirs me revenaient par vagues, les doigts de mon père qui griffaient la porte d'entrée pour me signifier son arrivée, ses grimaces quand il me mangeait les pieds tel le pire des prédateurs, et ses gloussements quand je tardais à trouver sa cachette. ${ }^{153}$

Dès lors, les sons issus des actions de «glousser, griffer » servent d'appui de proximité avec la mort, et les sens (ouïe, odorat) remplacent le vide de la séparation et recréent la relation avec le défunt. De même, le bruit de la montre du père ravive les émotions liées à sa présence: «sa montre en argent émit un tintement sonore qui me serra le cœur. ${ }^{154}$.

Les multiples sensations de la fille font écho à ce que Marcel Proust a souligné à propos de la mémoire sensorielle:

«Mais, quand d'un passé ancien rien ne subsiste, après la mort des êtres, après la destruction des choses, seules, plus frêles mais plus vivaces, plus immatérielles, plus persistantes, plus fidèles, l'odeur et la saveur restent encore longtemps, comme des âmes, à se rappeler, à attendre, à espérer, sur la ruine de tout le reste, à porter sans fléchir, sur leur gouttelette presque impalpable, l'édifice immense du souvenir." 155

Le deuil se transforme ainsi en une période de réconfort, qui recourt de façon imprévue aux souvenirs des actes paternels et qui devient également un agent de distraction invitant la fille

(Le deuil dans Muettes de Yasmine Ghata ...) Dr. Aliaa Abdel Wahed 
endeuillée à s'éloigner de l'angoisse et implantant la figure du père.

En effet, les réminiscences s'enracinent dans le réel par l'évocation des gestes et des émotions qui les accompagnent. La mémoire devient émotionnelle et concrète. La narratrice décrit ses sentiments ainsi :

«Me sont revenues aussi la texture de sa peau tout juste rasée, son eau de Cologne capiteuse qui parfumait sa calvitie et la gymnastique de sa bouche quand il tentait d'allumer sa pipe ; le briquet incliné avait brûlé la peau de son index, une peau dure et rugueuse devenue insensible à la douleur. Ses bras puissants me protégeaient du monde. ${ }^{156}$

Les différents sens mobilisés par les termes comme : texture, parfumer, brûlure, rugueuse, sont suivis d'émotions comme : capiteuse, dure, douleur, puissant et protéger, qui révèlent, dans l'ordre, l'admiration de la fille pour l'eau parfumée de son père, la sensation de douleur générée par la brûlure, la protection du père, et le sentiment de sécurité qu'elle éprouvait lorsqu'il la prenait dans ses bras. Par conséquent, le deuil devient une période de régénération de mort au lieu d'être une manifestation de l'absence.

Par ailleurs, les odeurs lui permettent de vivre un état intérieur particulier, qu'elle ne peut plus ressentir que par l'odorat, ce qui évoque chez elle une mémoire émotionnelle. :

«Le seul fait que mon père ait pu voir, toucher ces papiers, en faisait des documents d'exception. Je humais les angles des pages,

(Le deuil dans Muettes de Yasmine Ghata ...) Dr. Aliaa Abdel Wahed 
certaine que ses empreintes y reposaient encore, lisais les commentaires rédigés de sa main. ${ }^{157}$

Le deuil est ainsi vécu d'une manière esthétique, et fait plaisir à la fillette endeuillée à travers ces signaux de perception qui accompagnent l'image du père, l'aidant à se le rappeler dans un plus large contexte:

«L'odeur de cardamone et de marc séché symbolisait cet orient méconnu, ses souks et ses rues. ${ }^{158}$.

Ce sont les lieux qu'elle a visités avec lui : elle passe des heures à humer les différentes odeurs qui évoquent des souvenirs visuels :

«Des heures entières à flairer ses traces. L'odeur de sa pipe avait embaumé sa collection de timbres. ${ }^{159}$

En outre, la mémoire du père est ressuscitée grâce au sens du toucher à travers lequel les scènes qu'elle a vécues avec lui s'animent devant elle.

«Il me suffisait d'en effleurer les creux et les saillies pour imaginer le Bosphore dans son lit, les collines alentour et les petites îles que mes ongles emportaient au large. Mes doigts glissaient et les vagues précipitaient tout sur leur passage. Refermer le répertoire sur cette page plongeait Istanbul dans une nuit étoilée. ${ }^{160}$

Le deuil s'accompagne plus facilement d'une mémoire involontaire avec le père et s'achève sur un mode nouveau, intériorisé, par la présence du disparu.

(Le deuil dans Muettes de Yasmine Ghata ...) Dr. Aliaa Abdel Wahed 
La mémoire visuelle donne également accès à la vie passée du père. : «Peu à peu, j'ajoutais à la musique l'examen systématique des livres d'art de mon père. Leurs illustrations me rappelaient des choses déjà vues; le clair-obscur des minarets d'Istanbul, les toitures de Topkapi et les revêtements en céramique ravivaient des souvenirs d'une autre vie, de la sienne. ${ }^{161}$

Dès lors, les lieux visités et les places qu'il affectionnait sont évoqués à la vue des images: "Mémoire visuelle qu'il me légua. Les images planes retrouvaient leur relief d'origine. Il me semblait effleurer du doigt les carreaux de la mosquée Bleue. A son insu, la glaçure de la page me livrait toutes les aspérités de la surface, les accidents de cuisson ou les angles poreux injuriés par le temps. ${ }^{162}$

En fait, les différents sens de perception ne sont qu'un avatar de ce puissant désir de la fille d'inscrire dans une forme matérielle la configuration définitive de son père. Les traces laissées ravivent les sensations et ressuscitent les actes enfouis.

«J'ai récupéré ses affaires de toilette. Une lotion pour cheveux à l'eau de Cologne me rappelait l'agilité de ses doigts. Comme lui, revisser et dévisser le bouchon d'un coup sec, le poser sur le rebord du lavabo ${ }^{163}$

Le deuil mémoriel s'appuie sur les apports sensoriels afin de régénérer des impressions, des sensations qui déclenchent le plaisir du vécu.

(Le deuil dans Muettes de Yasmine Ghata ...) Dr. Aliaa Abdel Wahed 
À force de recourir à la mémoire sensorielle et émotionnelle, le travail du deuil produit une ultime rencontre virtuelle avec l'autre. La fille garde un lien fort avec son père. Ainsi, la mémoire devient un lieu de présence, et matérialise les contours du visage du père invisible.

«je le voyais assis à côté de moi regardant défiler avec nostalgie les grandes dates de sa vie. L'ongle de son index posé sur une vieille photographie le localisait derrière des rangs de têtes étrangères. " ${ }^{164}$

Le moi en deuil parvient à survivre à la perte, à assumer son chagrin. Les propos de Jean Florence reste à ce discours significateurs :

«Une identification partielle achève alors le travail du deuil ; le moi en sort enrichi et l'objet, en quelque sorte, sauvé. Ne pourraiton pas considérer ce travail du deuil comme le type le plus général d'élaboration psychique d'un conflit ?... Le conflit du deuil réside dans l'antagonisme de l'amour, douloureusement fixé aux traces du disparu par la défaillance de l'objet qui brutalement fait défaut ${ }^{165}$

Dès lors, le deuil n'est plus une période d'angoisse, puisque la narratrice vainc la rupture de toute communication en se mettant en contact avec l'absent et en s'incorporant à lui.

«J'aimais reproduire ses gestes comme pour le faire apparaittre. Contrefaire son âme pour tromper la mienne. » ${ }^{166}$ 
Il semble donc être une reviviscence, un lieu de retrouvailles, à tel point qu'il devient une obsession, une manière de s'accommoder de la perte.

«Les livres gisaient à terre, je récupérais la moisson de ses yeux, les fauches de ses mains et les odeurs mêlées de ses voyages. ${ }^{167}$

Refaire les gestes de son père en s'intégrant à lui représente un palier protecteur afin de ne pas souffrir du manque de l'être cher.

«Moi, je faisais souvent le même rêve, j'avais beau me concentrer pour ne pas le faire, il revenait. La silhouette de mon père de moins en moins facile à reconstituer avec le temps, recroquevillée dans une malle en bois que je cachais, le cœur haletant comme si je lui sauvais la vie. J'arrangeais son visage, sa peau noircie, gommais l'usure avec une facilité déconcertante. ${ }^{168}$

En ce sens, le deuil mémoriel cède la place à la reconstruction imaginaire de l'être aimé perdu, entreprise dans la configuration de l'image du père. Ce processus d'incorporation a pour effet de maintenir vivant le disparu et de le faire advenir.

« C'est donc facile de réparer un mort, pas besoin d'instruments sophistiqués. C'est au réveil que la déception était grande, pas de malle, ni de maquillage, ni lui ni personne à protéger d'un ennemi. ${ }^{169}$, déclare la narratrice.

La fille porte en elle sa trace grâce à un retour imagé qui donne satisfaction, et grâce à des souvenirs qui témoignent d'une complétude. Cette stratégie d'identification au père permet à la 
fille de pallier son absence lorsqu'elle imagine qu'elle le porte en elle et le conserve.

«Au croisement de la question de la trace, de la question du langage et de celle du deuil, sont apparus sous un tout autre jour le concept de «substitution de l'objet perdu» et l'idée que «l'existence de l'objet perdu se poursuit psychiquement»» ${ }^{170}$

Le deuil est transfiguré sous la forme d'une figuration corporelle engagée dans l'expression physique de la narratrice.

«Je dormais sur le côté le plus affaissé, celui où mon père s'était allongé pendant des années. ${ }^{171}$

Elle s'imagine à la place où il s'allongeait auparavant sur le lit. Elle maintient ainsi sa présence par le recours aux mécanismes d'intériorisation.

«L'incorporation témoigne donc, à certains égards, que le sujet endeuillé ne peut momentanément se résoudre à perdre, abandonner le défunt, qu'il a besoin de maintenir celui-ci en vie pour se sustenter voire exister lui-même, à d'autres égards l'incorporation est bien la solution « de vie » cette fois que trouve inconsciemment le sujet pour actualiser, rendre présente - et se rendre présent à lui, « au présent de son Moi » ${ }^{172}$

«Le deuil, c'est finalement un travail qui consiste à reconnaître que l'autre est mort en dehors de nous, et qu'il est vivant en dedans de nous... Le deuil, c'est savoir deux choses en même temps : il n'est pas là mais il est en moi»» ${ }^{173}$ 
Elle se met à sa place afin de ressentir son existence, réagit de la même façon que lui, et se souvient même de ses réactions.

«La musique était ce que nous apprécions le plus, je sentais ses paupières s'entrouvrir au début d'un morceau. » ${ }^{174}$

Le père disparu est généré par cette identification à lui et par le fait que la narratrice se remette dans la peau de cet être qui lui était cher.

«Fascinée, je recopiais les ligatures de ses lettres sur une page vierge, sûre que son écriture deviendrait mienne à force de l'imiter. ${ }^{175}$

S'incorporer dans la peau du disparu devient une source essentielle de sa survie afin de concrétiser sa présence en l'imitant. Dans ce sens, Elle explique sa mode de vie à ses mots : «Elle est un geste, une forme. Elle est lui. » ${ }^{176}$

ce qui procure de nouveau sens au processus de deuil en intervenant le défunt au sein du quotidien pour garantir le sentiment de sécurité.

«J'avais pour mission de prolonger les plaisirs de mon père sur terre. Chaque jour, je lui accordais du temps pour raviver ses sens, ressusciter sa perception des choses. Sa propre mort n'était pas parvenue à éteindre son amour de l'art. » ${ }^{177}$

Dès lors, le travail de deuil correspond à la création d'un monde où survit son père, permettant d'intégrer en soi-même l'image de l'absent. Le deuil s'apparente à un combat contre la négativité de la séparation, à une invitation à retrouver l'être cher décédé.

(Le deuil dans Muettes de Yasmine Ghata ...) Dr. Aliaa Abdel Wahed 
«L'attention recueilli de mon père était parfois dissipée par les allées et venues de ma mère. Je sentais son regard glisser vers elle, s'égarer dans ses courbes. Mouvements brusques qui éprouvaient ses yeux si faibles. ${ }^{178}$

Dans ce sens, il est vécu comme une volonté de garder une composante affective et imagée, de recréer les moments passés, et de consolider le processus de la mémorisation.

En fait, la narratrice revoit son père fantasmé, elle décrit ainsi :

«Il les refermait, les formes se délaçaient en moi-même; je sentais quand il me déserter, surtout à la tombée de la nuit. Je laissais son corps modelé par le vide se libérer de nous. La plénitude de cette forme traversait la rue, secouait les branches des marronniers avant de disparaître dans l'immensité. ${ }^{179}$

À force de le mémoriser, elle le perçoit comme existant, venant à la maison et en repartant. Cependant, elle le revoit volontairement à chaque moment de la journée.

«L'appeler suffisait à le faire venir, une seule prière murmurée en moi-même me le ramenait. Il dirigeait mes pas au gré de ses humeurs, (...). » ${ }^{180}$

Dorénavant, la fille endeuillée se confronte à toutes les émotions avec son père mort. Le deuil renforce ainsi une réconciliation avec la vie, où l'absence extérieure est compensée par une présence intérieure.

«Mon père guida mes mains dans le répertoire et me souffla l'indicatif du pays. Le cœur haletant, je visualisais un câble

(Le deuil dans Muettes de Yasmine Ghata ...) Dr. Aliaa Abdel Wahed 
téléphonique sous l'océan bravant les profondeurs en un temps éclair, j'imaginais cette sonnerie brisant la quiétude de l'appartement avec vue sur le Bosphore. Trois sonneries et une voix suspendue dans le vide qui articula péniblement : «Merhaba ». Le cœur battant, je n'ai rien pu dire, j'ai entendu le bruit d'un klaxon sous sa fenêtre et la tonalité qui a interrompu notre silence anonyme. Papa mis ses mains sur les miennes pour replacer le combiné et pris congé de moi. ${ }^{181}$

Les réactions de la narratrice mettent en évidence son contact direct avec le défunt. Le deuil constitue, pour elle, une inspiration et un vecteur de créativité qui lui permettent de rendre hommage à son père disparu tout en véhiculant les émotions qu'elle vit en lien avec lui.

«C'était ce que mon père préférait chez sa femme, et mes yeux continuaient de nourrir les siens de ce spectacle. ${ }^{182}$

la fille construit un monde interne avec son père qui reste actif dans son psychisme. il est figuré et tend d'exister grâce à l'incorporation qui constitue une résistance contre la mort.

«je foulais le sol à pas lent. (...). Mon père n'était plus accroupi sur ses talons, bras grands ouverts pour freiner ma course et me faire voler. ${ }^{183}$

le deuil est devenu une phase d'indentification avec le défunt pour déjouer la frustration et la position dépressive liées à la perte. «Selon Freud, le deuil consiste à porter l'autre en soi. Il n'y a plus de monde, c'est la fin du monde pour l'autre à sa mort, et

(Le deuil dans Muettes de Yasmine Ghata ...) Dr. Aliaa Abdel Wahed 
j'accueille en moi cette fin du monde, je dois porter l'autre et son monde, le monde en moi : introjection, intériorisation du souvenir.» ${ }^{184}$ L'absence s'estompe au profit de la mémoire.

Il en est de même des traces scripturaires laissées par le père de son vivant. Toute une vie se présente devant la narratrice via l'intériorisation des souvenirs. "l'immortalité du mort ne réside que dans l'imaginaire des vivants. $\gg{ }^{185}$

Les lettres font figure de reliquaires renfermant les restes matériels d'une présence. "Les caractères noirs étaient autant de prétextes pour penser, pour ne rien penser. (...). C'est dans les archives de mon père que je tuerais l'ennui. ${ }^{186}$

L'angoisse de l'abandon se dissipe en faveur du plaisir pris à la lecture des mots du père, alors que les textes mis au jour servent de cadre mémoire pour la fille endeuillée, où chaque document véhicule un sens, une valeur et une vie.

«Documents lus et relus comme si un secret y était enfermé, photographies du siècle dernier examinées avec fascination. Toutes ces personnes disparues avaient à mes yeux un prestige que les vivants n'avaient pas. J'idéalisais le passé, le pedigree d'une famille que je sentais dans ma chair, dans mes entrailles. ${ }^{187}$

Ces empreintes expriment une espérance de survie. «En quelques heures, il m'avait tout donné de lui-même. J'allais jusqu'à imiter son écriture, $(\ldots ..) .{ }^{188}$

(Le deuil dans Muettes de Yasmine Ghata ...) Dr. Aliaa Abdel Wahed 
En fait, les traces de l'écriture du père permettent de récapituler constamment les instants significatifs. Elles suivent le fluide du temps, et présentent une vie commémorative qui intensifie les émotions pour mieux les ressentir.

Au demeurant, le deuil mémoriel désinhibe, chez la fille, le vide pour créer du lien, simule la présence et l'attachement au père absent, lui permettant ainsi de garder celui qu'elle a perdu et de perpétuer son souvenir sans jamais l'oublier.

Le philosophe Roland Barthes souligne l'importance de la photographie comme support mémoriel, car elle porte des émotions et des gestes. Les photographies répètent constamment les moments et les personnes disparus. : «répète mécaniquement ce qui ne pourra jamais plus se répéter existentiellement ${ }^{189}$

En regardant les clichés pris, la fille peut immortaliser les instants vécus et ranimer le passé, alors que les plus récents gardent un caractère tangible de sa présence au milieu de ses confrères. Les albums photo du père deviennent une nécessité vitale pour prolonger le contact visuel avec lui.

«Je sortais de l'oubli ces personnages sans noms, aux sourires éphémères, ces réunions familiales non loin du Bosphore où parents et enfants s'endimanchaient sous l'objectif d'un photographe arménien qui estampait son nom sous chaque portrait. » 190

L'espace mnésique échappe à la décadence du temps, et le médium des photos permet son appropriation, le revêtant ainsi

(Le deuil dans Muettes de Yasmine Ghata ...) Dr. Aliaa Abdel Wahed 
d'une valeur émotionnelle. Devenus des objets de ressourcement, les albums archivés attestent des liens sociaux, même avec les personnes décédées, et libèrent la narratrice des temps refoulés:

«Les femmes, séparées du groupe des hommes, offraient leurs meilleures postures, la tête haute et le mollet au repos. Quand je refermais ces albums, leurs visages hantaient mon sommeil, l'oubli me terrorisait. Je compris bien plus tard que je les avais réveillés dans leur profond sommeil. Harceler les défunts était devenu ma prière du soir. » ${ }^{191}$

Les photos permettent de conserver une représentation matérielle de moments de jouissance non représentables et fugitifs. La scène vécue par le père est transfigurée dans la mémoire affective où se vivent les meilleurs moments.

En fait, la fille se réfère à tout support permettant la résurrection de l'être aimé : soit les objets, soit les albums, soit les écrits. Tous ces éléments contiennent le portrait de la personne défunte pour signifier sa «présence-absence ${ }^{192}$ :

«Enfant sage, mon répertoire de motifs s'étoffait d'images mouvantes que j'attrapais au fil de mes humeurs. ${ }^{193}$

Par ailleurs, la narratrice puise dans les incidents du quotidien pour évoquer les moments perdus. Les incendies déclenchés dans la forêt à côté de la maison sont transformés en source d'inspiration. La narratrice explique à ce propos :

«Cet incendie figurait les funérailles de mon père auxquelles je n'avais pas assisté. L'odeur de cendre et de terre mouillées

(Le deuil dans Muettes de Yasmine Ghata ...) Dr. Aliaa Abdel Wahed 


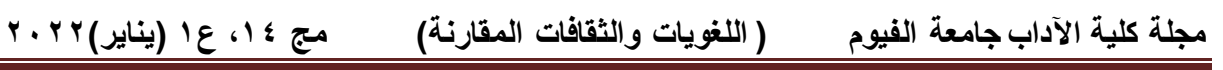

s'apparentait aux vapeurs d'encens. Son cercueil avait les contours nets des rivages de l'île Saint-Honorat, installé dans la nef centrale comme au large des côtes. » ${ }^{194}$

La fille opère une correspondance visuelle et réciproque entre le paysage vécu et le souvenir du père : incendie et funérailles, où le présent ressuscite le passé.

À chaque moment de sa vie, la narratrice regénère la présence de son père par les scènes quotidiennes:

«L'homme n'accepte jamais, purement et simplement, la perte. La représentation refoulée du disparu reste investie et capable de faire effet à n'importe quel moment dans le psychisme ${ }^{195}$

De surcroît, elle investit les paysages afin qu'ils servent d'élan pour donner vie à son père : ainsi, les vacances à la plage, où elle avait l'habitude d'aller avec lui, deviennent des lieux de souvenirs:

« Je nageais autrefois sur le dos de mon père, observant les profondeurs et m'agrippant à son cou quand l'eau était trop noire. Sa tête pour gouvernail, je lui signalais les directions à prendre, il m'amusait à désobéir à mes consignes. »196

Ici, le passé est inscrit dans la mer, le soleil et les paysages, et les images refoulées reviennent à la surface, afin de faire revivre la présence du père.

«C'est la mer qui a été la plus honnête avec moi, pas de mensonges ni de facéties pour me consoler de l'absence. ${ }^{197}$

(Le deuil dans Muettes de Yasmine Ghata ...) Dr. Aliaa Abdel Wahed 
Par conséquent, la narratrice s'arrange pour vivre le temps passé avec le défunt à travers sa réminiscence de la mer, un endroit que son père aimait fréquenter pendant l'été. Les scènes évoquées ont dès lors la capacité de la divertir :

«Dans l'ennui, je me sentais exister. abattre les heures sans bouger, mon corps immobile pour traverser les choses. ${ }^{198}$

Grâce à la remémoration des moments vécus avec lui, elle se déplace et passe son temps à travers lui : «Mon père était omniprésent mais visible, sans existence mais bien là pourtant. $\gg^{199}$

Aux yeux de la narratrice, ce lieu constitue un soulagement mais en même temps une remémoration fidèle et concrète d'événements passés.

«J'éprouvais une libération dans l'angoisse, un salut dans la détresse, comme si j'avais enfin les mots, les bons mots pour le dire : «Papa » recouvert par les ondes. » ${ }^{200}$

En outre, elle est consolée par les souvenirs des moments passés au bord de la mer.

«C'est le deuil impossible d'une mémoire qui voudrait pouvoir faire surgir l'objet lui-même en lieu et place de la remémoration. Retrouver non pas la trace, mais la chose même» ${ }^{201}$

Il est évident que le deuil perd sa notion de dégénérescence grâce à la mémoire qui classe cette période dans la perspective de continuer avec le père absent, et d'établir des instants privilégiés et essentiels avec le mort.

(Le deuil dans Muettes de Yasmine Ghata ...) Dr. Aliaa Abdel Wahed 
«Je devins fétichiste, je traquais les traces de mon père laissées un an plus tôt. Des cendres, une pipe encore garnie d'un tabac sans odeur, ses savates éventrées, celles qu'il portait à la plage, une semelle molle en cuir auréolée de sel de mer. ${ }^{202}$

La narratrice trouve alors une conciliation avec le paysage devenu métonymique d'un univers plus ample : celui de la relation avec son géniteur.

«(...), quitter la mer me déchirait. Mon père n'était pas loin de nous en ces lieux. Pas de doute, il était encore à mi-chemin entre le monde des vivants et celui des morts, territoire que j'avais situé sur l'île Saint-Honorat. ${ }^{203}$

L'île Saint-Honorat est sacrée grâce à son monastère. Elle est en même temps située entre deux rives, espace métonymique où le monde de son père est installé. Le paysage aimé tue l'angoisse de la mort et la destruction de soi:

« La mer me rendait mon père à l'instant où le soleil à l'aplomb de la terre brûle les rochers $»^{204}$

ce qui compense la perte et libère la fille endeuillée de l'angoisse exclusive du décès. L'amour et la dépendance de son père sont transcendées par des lieux sacrés symboles de l'espace paternel si cher à travers lequel elle trouve son bien-être.

«mon esprit arpentait cet espace clos, j'aimais vagabonder, observant le motif de l'intérieur. Mon imaginaire a mille fois ouvert la porte de cet appartement estival, profitant de l'ennui de mes après-midi pour explorer l'abandon. ${ }^{205}$

(Le deuil dans Muettes de Yasmine Ghata ...) Dr. Aliaa Abdel Wahed 


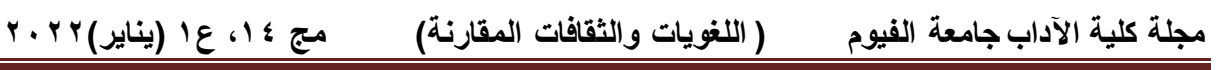

La mémoire demeure un travail du deuil accompli par les images et les sensations, et réalise la proximité vis-à-vis de l'absent, de ses émotions et de son expérience en vue de s'accommoder du quotidien.

«le processus du deuil est contemporain du travail de mémoire et de représentation, parfois de création sublimatoire, que le sujet mobilise face à la perte. ${ }^{206}$

Le deuil est une période investie et créative, qui remplace l'angoisse par le confort. Se rappeler le père devient un apaisement pour sa fille, Si l'être cher a disparu physiquement, le garder vivant mentalement est possible comme le souligne Bacqué :

« le travail du deuil se termine lorsque l' évocation de la personne décédée, par exemple le fait d' entendre son nom ou de penser à elle, n' entraine plus la détresse ${ }^{207}$ car il vit avec elle et en elle.

«Si l'être cher a disparu physiquement, on peut le garder vivant mentalement . Le deuil n'est plus signe de mort mais espoir d'exister éternellement ${ }^{208}$

(Le deuil dans Muettes de Yasmine Ghata ...) Dr. Aliaa Abdel Wahed 


\section{Conclusion}

Sous la plume de Yasmine Ghata, le deuil devient non plus une période macabre et triste, mais un mode de vie, un choix pour le vivant de se faire souffrir ou de se réjouir.

La recherche menée montre que l'on peut dépasser le chagrin du deuil sans recourir à la consolation d'autrui, si on a la faculté de gérer l'extrême affliction.

Le deuil devient alors une période d'investissement qui prend, dans cette étude, la voie de l'écriture ou de la mémoire.

Si l'écriture est un prolongement du monde endeuillé, elle n'aide pas l'écrivain à surmonter sa tristesse. Par conséquent, vivre le deuil sera plus pénible puisqu'il est dédoublé par l'écriture.

Quant au deuil investi par la mémoire, il est réussi si la personne endeuillée ravive et ressuscite, à son gré, la présence de l'être cher disparu. Le proche vivant déploie différents moyens comme l'imagination, les sens de la perception et l'incorporation, autant de facultés qui abolissent la disparition et qui aident à se réjouir de la présence du défunt.

Dans ce sens, le deuil transforme l'absence effective en une présence intérieure, et devient un processus subjectif destiné à maintenir vivant le défunt qui devient, en conséquence, la seule source de consolation pour la personne endeuillée avec qui il vit son quotidien.

En somme, le processus de deuil correspond à une régénération créative du défunt, à une révolte contre la perte, à un surcroît de

(Le deuil dans Muettes de Yasmine Ghata ...) Dr. Aliaa Abdel Wahed 


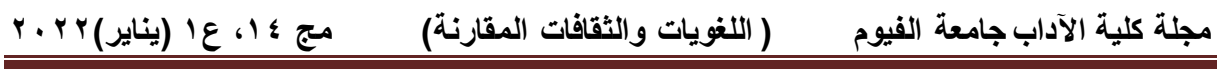

vie, à une richesse contre l'aporie de la mort, tout en étant aussi l'affirmation d'un lien continu avec la personne décédée.

«Chagrins comme tant de gens en éprouvent, et dont celui qui était actuellement ma torture ne se différenciait que par cette modalité du souvenir involontaire. ${ }^{209}$ 
${ }^{1}$ Yasmine Ghata, Muettes, Fayard, Paris, 2010

${ }^{1}$ Rosette Poletti, Barbara Dobbs-Zeller, Vivre le deuil en famille: des pistes pour traverser l'épreuve, Saint-Augustin, Suisse, 2001, p. 21

${ }^{3}$ Professeur des Universités, obtient le doctorat de psychologie clinique

${ }^{4}$ Maude Hélie, Le rôle de la spiritualité en tant que quête de sens dans l'adaptation au deuil: qu'en est-il des interventions psychothérapeutiques?, Thèse de doctorat en psychologie, Université du Québec, Canada, 2011 , p.10

5 Marie-Thérèse Bitsch, Pourquoi parler du deuil en 2015 ?, Revue Jusqu'à la mort accompagner la vie, Presses universitaires de Grenoble, Grenoble, Vol. 2, No. 121, 2015, p.11

${ }^{6}$ Ibid.

7 Vivre le deuil en famille: des pistes pour traverser l'épreuve, Op.cit. , p.25

${ }^{8}$ un écrivain et journaliste libertaire et suédois.

${ }^{9}$ Fréderic Jacquet, Naissances, Zeta Books, Bucarest, 2020, p.226

${ }^{10}$ Marie-Frédérique Bacqué, Apprivoiser la mort, Odile Jacob, Paris, 2003, p.9

11 Normand Doiron, Poétique de la consolation classique, Revue Dix-septième siècle, Presses Universitaires de France, Paris, Vol.4, No 237, 2007, p. 785

12 lbid.

13 Ibid.

${ }^{14}$ Marie-Frédérique Bacqué, Apprivoiser la mort, , Odile Jacob, paris, 2003 ; p14,

${ }^{15}$ Marie-Claude Leblanc-Beaulieu, le deuil en contexte nouveau. étude exploratoire de l'expérience de l'endeuillé lors de sa recherche de soutien en ligne, mémoire de la maîtrise en sciences des religions, Université du Québec, Montréal, 2006, p. 138

${ }^{16}$ Régine Scelles, Fratries confrontées au traumatisme, Presses universitaires de Rouen et du Havre, Rouen, 2009, p 127

17 Murielle Jacquet-Smailovic, Les conséquences d'un deuil dans l'enfance à moyen et à long terme, Revue internationale de soins palliatifs ,Médecine \& Hygiène, Swisse, Vol 26, No 1, 2011 p 17

${ }^{18}$ Muettes.Op.Cit. p.18

${ }^{19}$ Ibid. p. 14

${ }^{20}$ Ibid. p. 37

${ }^{21}$ Ibid. p. 11

${ }^{22}$ Flor María del Pilar Cifuentes, Le mal -être dans le deuil,: la place de l'art face à la précarité des rites dans les sociétés contemporaines, Thèse de Doctorat de Psychologie ,Université Paris 8 Vincennes, Paris, 2012 , p.106

${ }^{23}$ Muettes, Op.cit. p.12

${ }^{24}$ Emmanuelle Zech, Psychologie du deuil: impact et processus d'adaptation au décès d'un proche, Mardaga, Belgique, 2006, P61

${ }^{25}$ Muettes, Op.cit. p. 13

${ }^{26}$ Psychologie du deuil: impact et processus d'adaptation au décès d'un proche, Op.cit., p.62

${ }^{27}$ Muettes, Op.cit. p. 13

${ }^{28}$ Ibid.p.22

(Le deuil dans Muettes de Yasmine Ghata ...) Dr. Aliaa Abdel Wahed 
${ }^{29}$ Psychologie du deuil: impact et processus d'adaptation au décès d'un proche, Op.cit. p. 13

${ }^{30}$ Muettes, Op.cit. p.17

${ }^{31}$ Ibid.p.13

32 lbid.

${ }^{33}$ Psychologie du deuil: impact et processus d'adaptation au décès d'un proche, Op.cit. p.46

${ }^{34}$ Muettes. Op. Cit. p. 22

${ }^{35}$ Ibid.p.30

${ }^{36}$ Jeltje Gordon-Lennox, Funérailles: cérémonies surmesure, Labor et Fides, Genève, 2011, p. 13

${ }^{37}$ Muettes. Op. Cit. p. 17

${ }^{38}$ Chagnon, Joyce. Influence des rituels funéraires sur la résolution du deuil chez les personnes du troisième âge, Mémoire de Maîtrise en psychologie, Université du Québec à Trois-Rivières, Québec, Canada, 1994, P.17

${ }^{39}$ Muettes, Op.cit. p. 29

${ }^{40}$ Ibid.

${ }^{41}$ J. Kristeva, Soleil noir. Dépression et mélancolie, Paris, Gallimard, 1987, p. 75 ${ }^{42}$ Cf. Marie-Frédérique Bacqué, Deuil et santé, Odile Jacob, Paris, 1997, p. 22

${ }^{43}$ Muettes. Op. Cit. p. 18

44 Ibid.

45 lbid.

46 lbid.

${ }^{47}$ lbid. p. 22

48 lbid. p. 46

${ }^{49}$ Bérengère Beauquier- Maccotta,(Psychopathe) Le deuil périnatal: Clinique, pratiques et dispositifs thérapeutiques, Elsevier Health Sciences,

Amsterdam,2020, p. 102

${ }^{50}$ Muettes, Op.cit. p. 33

51 Ibid. p. 36

${ }^{52}$ Muettes, Op.cit. p. 22

${ }^{53}$ Muettes, Op.cit. p. 30

${ }^{54}$ Muettes, Op.cit. p. 20

${ }^{55}$ Emmanuelle Zech, Psychologie du deuil: impact et processus d'adaptation au décès d'un proche, Mardaga, Belgique, 2006, p. 62

${ }_{57}^{56}$ Muettes, Op.Cit. p.30

57 Ibid. p. 39

58 lbid. p. 35

${ }^{59}$ Muettes. Op.cit. p. 16

${ }^{60}$ Muettes. Op.cit. p. 15

61 lbid.

${ }^{62}$ Muettes. Op. Cit. p. 27

${ }^{63}$ Muettes. Op.Cit. p. 21

(Le deuil dans Muettes de Yasmine Ghata ...) Dr. Aliaa Abdel Wahed 
${ }^{64}$ Sigmund Freud, Deuil et Mélancolie, Revue Métapsychologie, Gallimard, Paris, 1968, pp. 146-147

${ }^{65}$ Muettes, Op.cit. p. 66

${ }^{66}$ Ibid. p. 68

${ }^{67}$ Prescendi, Francesca . Le deuil à Rome : mise en scène d'une émotion, Revue de l'histoire et des Religions, No.2, Colin, Paris, 2008, p. 308

${ }^{68}$ Muettes, Op.cit. p. 69

${ }^{69}$ Bourgeois, Marc-Louis. Deuil normal, deuil pathologique: clinique et psychopathologie, Doin, Rueil-Malmais on,2003, p.1

${ }^{70}$ Is abelle Auray, Processus de deuil, Proche Thiers, Thèse de Doctorat en psychologie, Université Lumière Lyon II, Lyon, 2016, P.139.

${ }_{71}^{71}$ Marie- Frédérique Bacqué, le Deuil à vivre, Odile Jacob, Paris, 1992, p.87

72 Jeanette Den Toonder, Voyages intérieurs dans trois romans contemporains. L'écriture intimiste de Bruno Hébert, Gaétan Soucy et Marie Laberge, Revue Globe, Volume 3, numéro 1, Québec, Canada, 2000, p.74

${ }^{73}$ Muettes, Op.cit. p 30

${ }^{74}$ Nicolas Lévesque, Le Deuil impossible nécessaire essais de la psychanalyse sur ta perte, ta mémoire et la culture, Thèse de Doctorat, en psychologie, université Montréal, canada, 2003, p. 32

${ }^{75}$ Thierno BAH, La Transition cédant-repreneur : une approche parla théorie $d u$ deuil, Revue française de gestion, Université Montpellier1, 2006, p.12

${ }^{76}$ Muettes, Op. Cit.p. 11

${ }^{77}$ Ibid

${ }^{78}$ Ibid. p. 22

${ }^{79}$ Ibid. p. 74

${ }^{80}$ Ibid. p. 12

${ }^{81}$ Ibid.p. 17

${ }^{82}$ Ibid. p.72

${ }^{83}$ Ibid. p. 30

${ }^{84}$ Ibid.p 72

${ }^{85}$ Ibid. p. 19

${ }^{86}$ Sara Garneau, La nuit comme un visage suivi de Paradoxes de l'écriture du deuil dans La plus que vive et Noireclaire de Christian Bobin, Maîtrise en études littéraires, Université Laval, Québec, Canada, 2020, p.120

${ }^{87}$ Ibid. p. 116.

${ }^{88}$ Muettes, Op. Cit.p. 11

${ }^{89}$ Havercroft, Barbara. (Poétesse et littéraire) Les traces vivantes de la perte, La poétique du deuil chez Denise Desautels et Laure Adler, Université du Québec à Montréal, Revue Voix et Images, Volume 36, No. 1, 2010, Canada, P.88

${ }^{90}$ Ibid. p.12

${ }^{91}$ Ibid.

92 Dominique Rabaté, Deuil et Littérature, Presses universitaire de Bordeaux, Pessac, 2005, p. 12

${ }^{93}$ Cf. Julia Kristeva, Soleil noir. Dépression et mélancolie, Gallimard, Paris, 1987, p78

${ }^{94}$ Muettes, Op. Cit. p 67 
95 Ibid, p. 25

${ }^{96}$ Ibid. p. 11

97 Ibid. p. 12

98 Bacqué, Marie-Frédérique. L'un sans l'autre. psychologie du deuil et des séparations, Larousse, Paris, 2007, p.17

${ }^{99}$ Muettes, Op. Cit.p.35

${ }^{100}$ Ibid.p. 74

${ }^{101}$ Ibid. p.56

102 Florence Gaiotti, Expériences de la parole dans la littérature de jeunesse contemporaine, Presses universitaires de Rennes, Rennes, 2009, p.257

${ }^{103}$ Muettes, Op. Cit. p. 35

${ }^{104}$ Ibid. p. 32

105 Ibid. p. 36

${ }^{106}$ Ibid. p. 21

${ }^{107}$ Ibid. p. 31

108 Kristeva, Julia. Soleil Noir, Dépression et mélancolie, Gallimard, Paris, 1987, p. 48 .

${ }^{109}$ Muettes, Op.cit., p. 77

${ }_{110}^{110}$ Muettes, Op.cit. p. 74

${ }^{111}$ Ibid.p. 78

112 Ibid. p. 76

113 Ibid. p. 75

${ }^{114}$ Ibid. p. 85

${ }^{115}$ Ibid. p. 85

${ }^{116}$ Ibid.p. 36

${ }^{117}$ Ibid. p. 62

${ }^{118}$ Ibid. p. 70

119 Ibid. p. 77

${ }^{120}$ Muettes, Op.cit. p. 75

121 Expériences de la parole dans la littérature de jeunesse contemporaine, Op.cit. p. 257

${ }^{122}$ Muettes, Op.cit. p. 12

123 Marie-Joëlle Savoie, De l'infini à l'œuvre dans le deuil, Deuil et dépression de Mélanie Klein, Revue Spirale, $\mathrm{n}^{\circ}$ 205, Conseil des Arts, Montréal, Canada, 2005 , p. 56

${ }^{124} \mathrm{Cf}$. Levesque, Nicolas. Le deuil impossible nécessaire, essais de psychanalyse sur ta perte, ta mémoire et la culture, Thèse de Doctorat en Psychologie, Université de Montréal, Québec, 2003, p. 32.

${ }^{125}$ Muettes, Op.Cit. p 14

${ }^{126}$ Ibid. p. 13

${ }^{127}$ Ibid. p. 20

${ }^{128}$ Ibid.

${ }^{129}$ Muettes, Op. Cit.p. 43

${ }^{130}$ Diana Kamienny Boczkowski, Le deuil, le corps, représentation, Revue de Psychanalyse, «Savoirs et clinique », Ères , Toulouse, volume 1, ${ }^{\circ} 10,2009$, p. 87

(Le deuil dans Muettes de Yasmine Ghata ...) Dr. Aliaa Abdel Wahed 
${ }^{131}$ Muettes, Op.cit. p. 18

${ }^{132}$ Marie Claire Lanctôt Bélanger, Vivre avec l'impermanence, Revue Spirale, La disparition, no 205, Conseil des arts et des lettres, Québec, Canada, 2005 , p. 17.

${ }^{133}$ Muettes, Op. Cit.p. 21

${ }^{134}$ Ibid. p. 44

${ }^{135}$ Ibid. p. 85

${ }^{136}$ Jean Lacan, «Variantes de la cure-type », Revue Écrits, , Seuil, Paris, 1966, p. 348

${ }^{137}$ Muettes. Op. Cit.p. 27

${ }^{138}$ Ibid. p. 48

${ }^{139}$ Ibid. p. 26

${ }^{140}$ Muettes, Op. Cit. p. 26

${ }^{141}$ Ibid.

${ }^{142}$ Ibid.

${ }^{143}$ Ibid. p. 21

${ }^{144}$ Ibid.

${ }^{145}$ Ibid. p. 27

${ }^{146}$ Ibid.

${ }^{147}$ Ibid. p. 30

${ }^{148}$ Ibid. p. 26

149 Ibid. p. 45

${ }^{150}$ Notons que la mémoire sensorielle ou perceptive, est liée aux cinq sens (vue, ouïe, odorat, goût et toucher). Elle sert à identifier des perceptions et renforce le lien entre le sujet et l'objet sans que l'individu en ait conscience

${ }^{151}$ Muettes, Op. Cit.p. 37

152 Ibid. p. 30

153 Ibid. p. 37

154 Ibid. p. 44

155 Proust, Marcel. A la recherche de temps perdu, Du côté de chez Swann, Gallimard, Paris , 1954, p 47

${ }^{156}$ Muettes, Ibid.p. 37

${ }^{157}$ Ibid. p. 44

${ }^{158}$ Ibid. p. 51

159 Ibid. p.44

160 Ibid. p. 51

${ }^{161}$ Ibid. p. 48

${ }^{162}$ Ibid. p. 49

163 Ibid. p. 61

${ }^{164}$ Ibid.p. 45

165 Jean Florence, L'identification dans la théorie freudienne, Publications des Facultés Universitaires, Saint-Louis, , Bruxelles, 1978, pp.288-289.

${ }^{166}$ Muettes, Op.cit. p. 49

${ }^{167} \mathrm{Ibid}$.

${ }^{168}$ Muettes, Op.cit. pp. 72-73

169 Ibid. 
170 Le deuil impossible nécessaire, essais de psychanalyse sur ta perte, ta mémoire et la culture, Op.cit. p. 12

${ }^{171}$ Muettes, Op.cit. p.18

${ }^{172}$ Roussillon René. Agonie, clivage et symbolisation, PUF, Paris,1999, p.143

${ }^{173}$ Nasio Juan - David. La douleur d'aimer. Paris, Payot, 2005, p. 140

${ }^{174}$ Muettes, Op.cit. p.47

175 Ibid. p. 44

${ }^{176}$ Ibid. p. 85

${ }^{177}$ Muettes, Op. Cit.p. 47

${ }^{178}$ Ibid. p. 48

179 Ibid.

${ }^{180}$ Ibid. p. 50

181 Ibid.p.51

182 Ibid.p.53

183 Ibid.p.57

${ }^{184}$ Marie Joël St-Louis Savoie, Chaque fois unique, la fin du monde: figures et apories du deuil selon Jacques Derrida, Thèse de Magistère, Université de Montréal, Québec, 2005, p. 16

${ }^{185}$ Christian Mormont, Deuil et Traumatisme, Revue Francophone, Stress et Trauma, Elsevier, Paris, Vo.1 9 no 4, 2009, p 222

${ }^{186}$ Muettes, Op.cit. p.43

187 Ibid. P. 27

${ }^{188}$ Ibid. P. 49

${ }^{189}$ Roland Barthes, La chambre claire. Note sur la photographie, , Seuil, Paris, 1980, p. 15

${ }^{190}$ Muettes, Op.cit.p. 27

${ }^{191}$ Ibid. p. 28

${ }^{192}$ Augustin Bado, Deuil et narration à partir du malade en phase terminale, Thèse de Doctorat en Philosophie, Université de Valencia, Italie, 2019, p235

${ }^{193}$ Muettes, Op. Cit.p. 21

${ }^{194}$ Ibid. p. 71

${ }^{195}$ Le deuil impossible nécessaire : essais de psychanalyse sur la perte, la mémoire et la culture, Op.cit. p. 30

${ }^{196}$ Muettes, Op. Cit. p. 58

${ }^{197}$ Ibid. p. 74

${ }^{198}$ Ibid. p. 20

199 Muettes, Op. Cit. p. 76

${ }^{200}$ Ibid. p. 63

${ }^{201}$ Dominique Scarfone, Oublier Freud?, Mémoire pour la psychanalyse, Boréal, Montréal, Canada,1999,p. 219

${ }^{202}$ Muettes, Op.cit. p. 61

${ }^{203}$ Ibid. p. 75

${ }^{204}$ Ibid. p. 76

${ }^{205}$ Ibid. p. 84

${ }^{206}$ le deuil, le corps, représentation, Op.cit.p. 22 


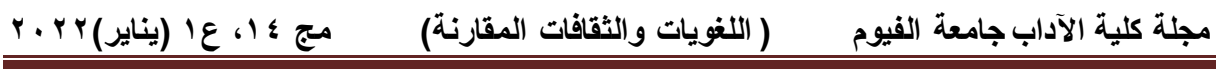

${ }^{207}$ Maude Hélie, Le rôle de la spiritualité en tant que quête de sens dans

l'adaptation au deuil: qu'en est-il des interventions psychothérapeutiques? ,

Thèse de Doctorat en psychologie, Université du Québec, Canada, 2011, p. 21.

${ }^{208}$ Ibid. p. p. 22.

${ }^{209}$ Sigmund Freud, Deuil et Mélancolie, PUF, Paris, 2005, p165

(Le deuil dans Muettes de Yasmine Ghata ...) Dr. Aliaa Abdel Wahed 


\section{Bibliographie}

\section{I - L'œuvre corpus}

Yasmine Ghata, Muettes, Fayard, Paris, 2010

\section{II - Ouvrages consultés}

Bacqué, Marie-Frédérique :

- L'un sans l'autre. psychologie du deuil et des séparations, Larousse,

Paris, 2007

- le Deuil à vivre, Odile Jacob, Paris, 1992

- Deuil et santé, Odile Jacob, 1997

- Apprivoiser la mort, Odile Jacob, Paris, 2003

- Bourgeois, Marc-Louis. Deuil normal, deuil pathologique: clinique et psychopathologie, Doin, Rueil-Malmaison,2003

- Barthes, Roland. La chambre claire. Note sur la photographie, Seuil, Paris, 1980

- Beauquier- Maccotta, Bérengère. Le deuil périnatal: Clinique, pratiques et dispositifs thérapeutiques, Elsevier Health Sciences, Amsterdam, 2020

- Freud, Sigmund. Deuil et Mélancolie, PUF, Paris, 2005

- Jacquet, Fréderic. Naissances, Zeta Books, Bucarest, 2020

- Gaiotti, Florence. Expériences de la parole dans la littérature de jeunesse contemporaine, Presses universitaires de Rennes, Rennes, 2009 ,

- Gordon-Lennox, Jeltje. Funérailles: cérémonies sur mesure, Labor et Fides, Genève, 2011

- Florence, Jean. L'identification dans la théorie freudienne, Publications des Facultés Universitaires, Saint-Louis, Bruxelles, 1978

- Kristeva, Julia. Soleil Noir, Dépression et mélancolie, Gallimard, Paris, 1987

- Nasio, Juan - David. La douleur d'aimer. Paris, Payot, 2005

- Poletti, Rosette. Dobbs-Zeller, Barbara. Vivre le deuil en famille: des pistes pour traverser l'épreuve, Saint-Augustin, Suisse, 2001

(Le deuil dans Muettes de Yasmine Ghata ...) Dr. Aliaa Abdel Wahed 
- Proust, Marcel. A la recherche de temps perdu, Du côté de chez Swann, Gallimard, Paris , 1954

- Rabaté, Dominique. Deuil et Littérature, Presses universitaire de Bordeaux, Pessac, 2005

- Roussillon, René. Agonie, clivage et symbolisation, PUF, Paris, 1999

-Scarfone, Dominique. Oublier Freud?, Mémoire pour la psychanalyse, Boréal, Montréal, Canada,1999

- Scelles, Régine. Fratries confrontées au traumatisme, Presses universitaires de Rouen et du Havre, Rouen, 2009

- Zech, Emmanuelle. Psychologie du deuil: impact et processus d'adaptation au décès d'un proche, Mardaga, Belgique, 2006

\section{III- Revues consultées}

- Bah, Thierno. La Transition cédant-repreneur : une approche par la théorie $d u$ deuil, Revue française de gestion, Université Montpellier1, 2006

- Bitsch, Marie-Thérèse. Pourquoi parler du deuil en 2015 ?, Revue Jusqu'à la mort accompagner la vie, Presses universitaires de Grenoble, Vol. 2, No. 121, 2015, p.11

- Den Toonder, Jeanette. Voyages intérieurs dans trois romans contemporains. L'écriture intimiste de Bruno Hébert, Gaétan Soucy et Marie Laberge, Revue Globe, Volume 3, numéro 1, Québec, Canada, 2000,

- Doiron, Normand. Poétique de la consolation classique, Revue Dixseptième siècle, Presses Universitaires de France, Vol.4, No 237, 2007

- Freud, Sigmund. Deuil et Mélancolie, Revue Métapsychologie, Gallimard, Paris, 1968

- Jacquet-Smailovic, Murielle. Les conséquences d'un deuil dans l'enfance à moyen et à long terme Revue internationale de soins palliatifs , Médecine \& Hygiène, Suisse, Vol 26, No 1, 2011

-Kamienny Boczkowski, Diana. Le deuil, le corps, représentation, Revue de Psychanalyse, "Savoirs et clinique », Ères , Toulouse, volume $1, \mathrm{n}^{\circ}$ 10, 2009

- Lacan, Jean. «Variantes de la cure-type », Revue Écrits, Seuil, Paris, 1966

(Le deuil dans Muettes de Yasmine Ghata ...) Dr. Aliaa Abdel Wahed 
- Lanctôt Bélanger, Marie Claire .Vivre avec l'impermanence, Revue Spirale, La disparition, no 205, Conseil des arts et des lettres, Québec, Canada, 2005

- Mormont, Christian. Deuil et Traumatisme, Revue Francophone, Stress et Trauma, Elsevier, Paris, Vo.1 9 no 4, 2009

- Prescendi, Francesca. Le deuil à Rome : mise en scène d'une émotion, Revue de l'histoire et des Religions, No.2,Colin, Paris, 2008.

\section{IV-Thèses consultées}

- Auray, Isabelle. Processus de deuil, Proche Thiers, Thèse de Doctorat en Psychologie, Université Lumière Lyon II, Lyon, 2016

-Bado, Augustin. Deuil et narration à partir du malade en phase terminale, Thèse de Doctorat en Philosophie, Université de Valencia, Italie, 2019

- Chagnon, Joyce. Influence des rituels funéraires sur la résolution du deuil chez les personnes du troisième âge, Mémoire de Maîtrise en psychologie, Université du Québec à Trois-Rivières, Canada, 1994

- Garneau, Sara. La nuit comme un visage suivi de Paradoxes de l'écriture du deuil dans La plus que vive et Noireclaire de Christian Bobin, Maîtrise en études littéraires, Université Laval, Québec, Canada, 2020

- Hélie, Maude. Le rôle de la spiritualité en tant que quête de sens dans l'adaptation au deuil: qu'en est-il des interventions psychothérapeutiques?, Thèse de Doctorat en psychologie, Université du Québec, Canada, 2011

- Leblanc-Beaulieu, Marie-Claude .Le deuil en contexte nouveau. étude exploratoire de l'expérience de l'endeuillé lors de sa recherche de soutien en ligne, Mémoire de mattrise en sciences des religions, Université du Québec, Montréal, 2006

- Lévesque, Nicolas. Le Deuil impossible nécessaire essais de la psychanalyse sur ta perte, ta mémoire et la culture, Thèse de Doctorat, en psychologie, université Montréal, Canada, 2003

- St-Louis Savoie, Marie Joël. Chaque fois unique, la fin du monde: figures et apories du deuil selon Jacques Derrida, Thèse de Magistère, Université de Montréal, Québec, 2005

(Le deuil dans Muettes de Yasmine Ghata ...) Dr. Aliaa Abdel Wahed 
- Wilhelm, Bénédicte. Aide aux endeuillés par les équipes de soin palliatif, Thèse de Doctorat en médecine, Université Henri Poincaré, Nancy1, 2001

\section{V-Webographie}

1- Site Cairn, Baussant- Crenn, Camille. Le deuil : aspects cliniques, théoriques, thérapeutiques, Dans Accompagner des personnes en deuil (2004)

Repéré à : https://www.cairn.info/accompagner-des-personnes-endeuil--9782749202228-page-15.htm

2- Site Cairn: Bacqué, Marie-Frédérique, Qu'est-ce que le deuil ?, Dans Le deuil (2012)

Repéré à : https://www.cairn.info/le-deuil--9782130590774-page20.htm

3- Site Cairn: Bacqué, M.-F. \& Hanus, M. (2016). Le deuil (7e éd.). Paris, France : Presses universitaires de France. «Que sais-je ?».

Repéré à : http://www. cairn.info.ezproxy. usherbrooke.ca/le-deuil-9782130750215.htm

4- Site Persée Livre: Chantal de Gournay, Le deuil de l'image. De la photographie à l'image virtuelle, Réseaux. Communication Technologie - Société, No 61, 1993.

Repéré à :https $/ /$ www.persee.fr/doc/reso_07517971_1993_num_11_61_2408?q=deuil

5- Site Open Edition Journals : Prescendi, Francesca. «Le deuil à Rome: mise en scène d'une émotion », Revue de l'histoire des Religions en ligne, No.2, 2008.

Repéré à :URL : http://journals.openedition.org/rhr/6123 ;DOI : https://doi.org/10.4000/rhr.6123

6- Site Erudit, Havercroft, Barbara. Les traces vivantes de la perte La poétique du deuil chez Denise Desautels et Laure Adler, Université du Québec à Montréal, Volume 36, No 1, 2010.

Repéré à : https//www.erudit.org/fr/revues/vi/2010-v36-n1vi3972/045236ar.pdf

(Le deuil dans Muettes de Yasmine Ghata ...) Dr. Aliaa Abdel Wahed 


\section{الحداد في رواية البكماوات لياسمين غتا}

\section{ملخص}

يتتاول البحث موضوع الحداد و الذي هو مرحلة ما بعد وفاة الثخص الذي نحبه ،

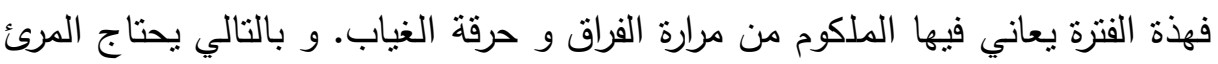

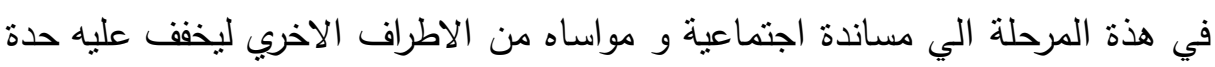

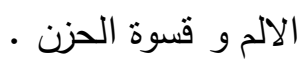

الا ان الثخصيات في رواية الكانبة اللبنانية ياسمين غتا يرفضون تلقي التعازي من المقربين و يريدون ان يتالموا في صمت و في في وحدة .

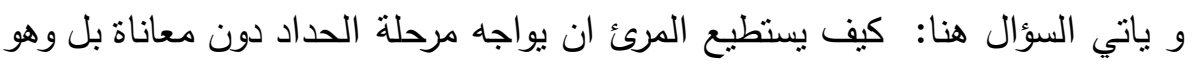

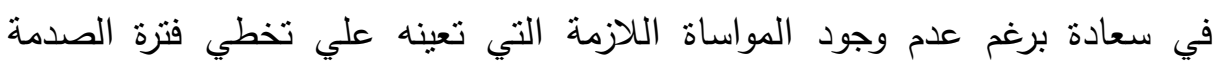
النفسية؟ و ينتاول البحث ثلاث نقاط اولها الحداد بلا مواساة و الثانية الحداد بالكتابة و الثالثة الحداد بالذاكرة ففي النقطة الاولي نكثف سلوك الام و ابنتها في معايشة الحداد دون مواساة و كيف استقبلوا صدمة موت الاب و كيف تعاملوا معها. ثم نري ان الحياة دون مواساة

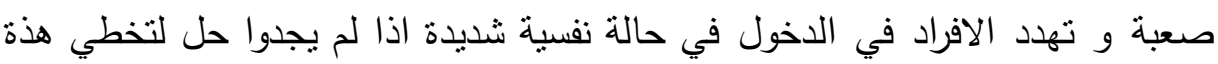
المرحلة. في النقطة الثانبة نعرض الوسيلة التي اتخذتها الام للخروج من حدادهاو هي الكتابة

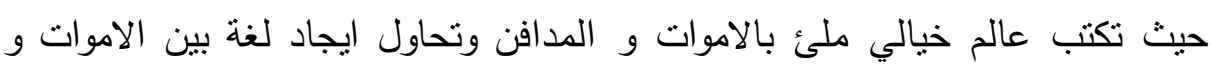
الاحياء، و لكن نجد الكتابة وسيلة مؤقتة لانها تتنهي بانتهاء الكتاب و كذللك ترسم

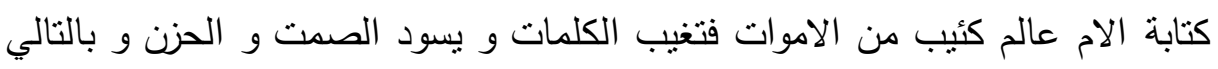
تعنبر الكتابة كتابة تطيل الحدادو تعرضه.

(Le deuil dans Muettes de Yasmine Ghata ...) Dr. Aliaa Abdel Wahed 


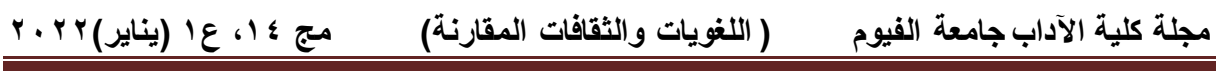

ثم نستعرض النقطة الثالثة و الاخيرة وهي الوسيلة التي انتهجتها الطفلة لتخطي فترة الحداد و هي التذكرة بالاب و كيفية انعاش الذاكرة باستمرار من خلال تاملها لمقتيات و اشياءالتي تركها الاب فتعيد لها ذكريات الماضي واستعادة الاحاسيس مع الاب من خلال الحواس الخمس ومحاولتها بالذكريات ادماج الاب بداخلها لنشعر باحاسيسه و حركاته كانه يعيش معها طول الوقت. و بالتالي يكتسب الحداد معني جديدا له فلا يكون دلالة عن الوفاة و لكن عن الاحياء و لم يعد ايضا يعبرعن الحزن و لكن عن متعة لقاء الفقيد الغالي. 